\title{
Simulating Many-Body Systems with a Projective Quantum Eigensolver
}

\author{
Nicholas H. Stair ${ }^{*}$ and Francesco A. Evangelista $\odot^{\dagger}$ \\ Department of Chemistry and Cherry Emerson Center for Scientific Computation, Emory University, Atlanta, \\ Georgia 30322, USA
}

(Received 2 February 2021; accepted 4 June 2021; published 1 July 2021)

\begin{abstract}
We present a new hybrid quantum-classical algorithm for optimizing unitary coupled-cluster (UCC) wave functions deemed the projective quantum eigensolver (PQE), amenable to near-term noisy quantum hardware. Contrary to variational quantum algorithms, PQE optimizes a trial state using residuals (projections of the Schrödinger equation) rather than energy gradients. We show that the residuals may be evaluated by simply measuring two energy expectation values per element. We also introduce a selected variant of PQE (SPQE) that uses an adaptive ansatz built from arbitrary-order particle-hole operators, offering an alternative to gradient-based selection procedures. PQE and SPQE are tested on a set of molecular systems covering both the weak and strong correlation regimes, including hydrogen clusters with four to ten atoms and the $\mathrm{BeH}_{2}$ molecule. When employing a fixed ansatz, we find that $\mathrm{PQE}$ can converge disentangled (factorized) UCC wave functions to essentially identical energies as variational optimization while requiring fewer computational resources. A comparison of SPQE and adaptive variational quantum algorithms shows that - for ansätze containing the same number of parameters - the two methods yield results of comparable accuracy. Finally, we show that for a target energy accuracy, SPQE provides a parameterization of similar size or more concise than the one obtained via selected configuration interaction and the density matrix renormalization group on one- to three-dimensional strongly correlated $\mathrm{H}_{10}$ systems in terms of number of variational parameters.
\end{abstract}

DOI: 10.1103/PRXQuantum.2.030301

\section{INTRODUCTION}

Efficient quantum algorithms for determining the ground and excited states of many-body systems are of fundamental interest to chemistry, condensed matter physics, and materials science [1-4]. The ability of quantum devices to represent $N$-body states with qubits scaling linearly in $N$ make them particularly appealing for representing highly entangled states, as is common in systems with strongly correlated electrons. Therefore, quantum (and hybrid quantum-classical) algorithms offer an alternative to methods such as the density matrix renormalization group [5] (DMRG), selected configuration interaction [6,7], determinant-based Monte Carlo [8], variants of coupled-cluster (CC) theory $[9,10]$ amenable to treating strong correlation [11-14], and multireference CC (MRCC) methods [15-20]. Although these classical

\footnotetext{
*nstair@emory.edu

${ }^{\dagger}$ francesco.evangelista@emory.edu
}

Published by the American Physical Society under the terms of the Creative Commons Attribution 4.0 International license. Further distribution of this work must maintain attribution to the author(s) and the published article's title, journal citation, and DOI. algorithms can accurately predicted energies and properties of certain classes of strongly correlated systems, they still have high-order polynomial or exponential cost in the general case.

Since Feynman's proposal to use a controlled quantum system to carry out simulations [21], significant algorithmic and experimental advances have been made. The earliest demonstrations of quantum simulation for small molecules [2] utilized the quantum phase estimation algorithm [22-24] with Suzuki-Trotter decomposed time evolution $[25,26]$ of an adiabatically prepared trial state. It is believed that some combination of these techniques will permit the efficient simulation [27-30] of certain classes of Hamiltonians [31], but that they may require deep circuits with high fidelity, a requirement incompatible with current noisy intermediate-scale quantum (NISQ) devices [32]. Several low-depth quantumclassical hybrid algorithms have been developed for NISQ hardware. These algorithms prepare and measure properties of many-body states on a quantum device, but they store and optimize the parameters that define such states on a classical computer. The variational quantum eigensolver (VQE) approach [33-36] has been used in several landmark experiments, demonstrating quantum calculations on nontrivial molecular systems [3,37-41]. In VQE, the ground state is approximated by a normalized trial 
state $|\tilde{\Psi}\rangle=\hat{U}(\mathbf{t})\left|\Phi_{0}\right\rangle$, in which the unitary operator $\hat{U}(\mathbf{t})$ depends on the parameter vector $\mathbf{t}$ and $\Phi_{0}$ is (usually) an unentangled reference state. The VQE energy $\left(E_{\mathrm{VQE}}\right)$ is then obtained by minimization of the trial state energy expectation value as

$$
E_{\mathrm{VQE}}=\min _{\mathbf{t}}\left\langle\Phi_{0}\left|\hat{U}^{\dagger}(\mathbf{t}) \hat{H} \hat{U}(\mathbf{t})\right| \Phi_{0}\right\rangle .
$$

The VQE scheme employs an optimization algorithm running on a classical computer to minimize the energy expectation value, with all inputs (energy and gradients) being evaluated with the help of a quantum computer. An important advantage of VQE over classical many-body methods is the ability to use trial states that cannot be represented efficiently on a classical computer. VQE was initially implemented with an exponential operator ansatz inspired by unitary coupled-cluster (UCC) theory [42-48], but has more recently been extended to hardware-efficient [3] and qubit-space [49] UCC variants as well. We exclusively use the abbreviation UCC to refer to unitary coupled-cluster theory, and not unrestricted formulations of conventional coupled-cluster methods [50], which historically share this abbreviation. Other promising hybrid approaches include quantum imaginary time evolution [51,52], and quantum subspace diagonalization techniques [51,53-56].

Despite the indisputable importance of VQE in the field of quantum simulation, there are a few drawbacks that make its practical application challenging to large-scale problems. One such issue is the slow convergence of VQE due to noise in the measured energy and gradients, and the large-scale nonlinear nature of the optimization problem. These issues are compounded by the sizable number of total measurements needed for operator averaging [57]. Another challenge is the potentially large number of classical parameters and resulting circuit depth necessary to predict sufficiently accurate energies. These two problems are likely exacerbated in systems with strongly correlated electrons.

Progress addressing these deficiencies of VQE has been made on several fronts. For example, grouping commuting Pauli operators $[3,35,58-60]$, utilizing integral factorization strategies [61], and employing alternative bases [29, 62] have been shown to reduce the number of measurements needed for operator averaging. Concurrently, computationally feasible approaches for measuring analytical gradients with quantum devices using the parametershift rule [63], or its recent lower-cost variant [64], have allowed gradient-based VQE to become potentially realizable on NISQ hardware. Other advances, of particular importance to this work, include VQE ansätze constructed iteratively, as done in the adaptive derivative-assembled pseudo-Trotterized VQE [36] (ADAPT VQE) and the iterative qubit coupled-cluster [65] (iQCC) methods. The primary advantage of ADAPT VQE and $\mathrm{QQCC}$ is their ability to produce compact ansätze that result in fewer classical parameters, and shallower quantum circuits than those from UCC truncated to a given particle-hole rank. However, these advantages come at the cost of a greater number of energy and gradient evaluations for optimizing and selecting new unitary operators. Investigating more efficient ways to select important operators is an ongoing area of research $[66,67]$.

In this work, we present an alternative to $\mathrm{VQE}$ for optimizing the amplitudes of a factorized form of the UCC ansatz (often referred to as Trotterized [35] or quantum [68] UCC), given by a product of exponential operators rather than the exponential of a sum of operators. We refer to this ansatz as disentangled UCC (dUCC) - a terminology borrowed from the field of Lie theory-to reflect the fact that it is not an approximation of UCC [69]. Inspired by the projective approach used in classical coupled-cluster theory $[10,15]$, we propose an alternative trial state optimization algorithm that we deem the projective quantum eigensolver (PQE). PQE does not rely on variational minimization and therefore does not need energy gradients. Instead, PQE requires only the evaluation of residuals, that is, projections of the Schrödinger equations onto a linearly independent basis. As shown in this paper, residuals may be easily measured on NISQ devices with similar or fewer measurements than analytical gradients, and require quantum circuits that contain only one additional exponential term. We also propose a new selection scheme for identifying important operators based on the residual vector. This selected variant of PQE (SPQE) requires no predefined operator pool and employs only a small number of measurements to identify important operators. To demonstrate the practical advantages of PQE, we perform a comparison of VQE and PQE using a fixed dUCC ansatz for several molecular systems in the regime of weak and strong correlation, also considering the effect of stochastic noise. Finally, we compare SPQE against the ADAPT VQE approach, selected configuration interaction, and the density matrix renormalization group.

\section{THEORY}

\section{A. The projective quantum eigensolver}

In this work, we propose to obtain the ground state of a general many-body system using a projective approach. Like in VQE, we approximate the ground state using a trial state $|\tilde{\Psi}(\mathbf{t})\rangle=\hat{U}(\mathbf{t})\left|\Phi_{0}\right\rangle$. After inserting the definition of the trial state in the Schrödinger equation and left multiplying by $\hat{U}^{\dagger}(\mathbf{t})$, we obtain the condition

$$
\hat{U}^{\dagger}(\mathbf{t}) \hat{H} \hat{U}(\mathbf{t})\left|\Phi_{0}\right\rangle=E\left|\Phi_{0}\right\rangle .
$$

Projection onto the reference state $\Phi_{0}$ yields the PQE energy

$$
E_{\mathrm{PQE}}(\mathbf{t})=\left\langle\Phi_{0}\left|\hat{U}^{\dagger}(\mathbf{t}) \hat{H} \hat{U}(\mathbf{t})\right| \Phi_{0}\right\rangle,
$$


a quantity that is still an upper bound to the exact ground state energy. Projections onto the complete set of orthonormal many-body basis functions complementary to $\Phi_{0}$, here denoted as $Q=\left\{\Phi_{\mu}\right\}$, yields a set of residual conditions

$$
r_{\mu}(\mathbf{t}) \equiv\left\langle\Phi_{\mu}\left|\hat{U}^{\dagger}(\mathbf{t}) \hat{H} \hat{U}(\mathbf{t})\right| \Phi_{0}\right\rangle=0 \quad \text { for all } \Phi_{\mu} \in Q
$$

where $r_{\mu}$ is an element of the residual vector and $\mu$ runs over all elements of the many-body basis. Equations (3) and (4) form a system of nonlinear equations in the parameter vector $\mathbf{t}$ that may be solved via a classical iterative solver. For an approximate ansatz with the number of parameters less than the dimension of the $Q$ space, Eq. (4) can be enforced only for a subset of the residuals. Then, the complete projection space $Q$ can be partitioned into two sets: (i) $R$, the space of basis functions $\Phi_{\mu}$ for which $r_{\mu}=0$ is enforced, and (ii) $S=Q \backslash R$, the complementary space for which $r_{\mu}$ may not be null.

Figure 1 illustrates the connection between the PQE residual condition and the uncertainty in the ground-state energy estimated via Eq. (3). By the Gershgorin circle theorem, the difference between the exact $(E)$ and the PQE $\left(E_{\mathrm{PQE}}\right)$ ground-state energy, $\left|E_{\mathrm{PQE}}-E\right|$, is bound by the

(b) Residual vector

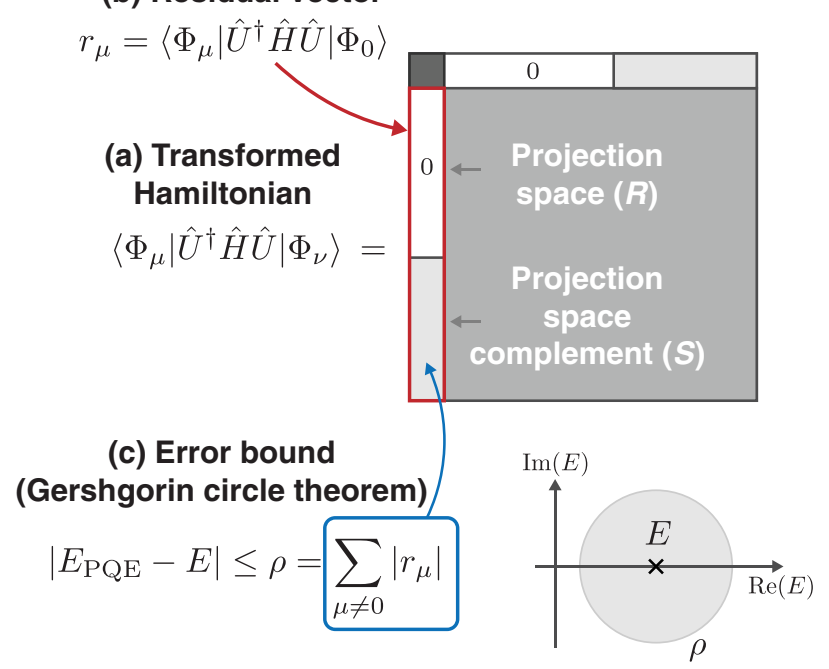

FIG. 1. Connection between the norm of the PQE residual and the energy error via the Gershgorin circle theorem. (a) Structure of the transformed Hamiltonian in the basis of orthogonal states $\left\{\Phi_{\mu}\right\}$. (b) The residual vector is the first column of the transformed Hamiltonian matrix (first element excluded). (c) The difference between the approximate ground-state PQE energy $\left(E_{\mathrm{PQE}}\right)$ and the exact eigenvalue $(E)$ is bound by the radius $\rho$, which is equal to the 1-norm of the residual vector. The part of $r_{\mu}$ that corresponds to states in the projection manifold $R$ is null for the PQE solution, while elements involving projections on the space $S=Q \backslash R$ are generally nonzero and determine the value of $\rho$. radius $\rho=\sum_{\mu \neq 0}\left|r_{\mu}\right|$, where $\mu$ runs over the entire manybody basis, excluding the reference determinant. Therefore, when the residual is null $(\rho=0)$, the PQE energy is exact. When the PQE equation is satisfied only by a subset of the many-body basis - as in the case of an approximate trial state - the error $\left|E_{\mathrm{PQE}}-E\right|$ is bound by the sum of the absolute value of the residual elements $\left|r_{v}\right|$ with $\Phi_{v} \in S$, for which the PQE equation is not satisfied.

Note that the residual condition [Eq. (4)] is satisfied by any eigenstate, and that the Gershgorin circle theorem error bound also applies to excited states. A potential disadvantage is that $\mathrm{PQE}$ could converge on an excited state (an issue we did not experience in this study). However, this feature could be used to formulate excited state algorithms based on PQE, which use the residual condition as a criterion for convergence and do not require costly measurement of the variance, as is commonly done in VQE [35].

The PQE is a general approach; however, in the following, we focus on its applications to interacting fermions using a disentangled (factorized) form of the unitary coupled-cluster ansatz. We assume that our system is described by the general two-body Hamiltonian

$$
\hat{H}=\sum_{p q} h_{p q} \hat{a}_{p}^{\dagger} \hat{a}_{q}+\frac{1}{4} \sum_{p q r s} v_{p q r s} \hat{a}_{p}^{\dagger} \hat{a}_{q}^{\dagger} \hat{a}_{s} \hat{a}_{r},
$$

where $\hat{a}_{p}\left(\hat{a}_{q}^{\dagger}\right)$ is a fermionic annihilation (creation) operator, while $h_{p q}$ and $v_{p q r s}$ are one-electron and antisymmetrized two-electron integrals, respectively [70].

\section{B. Traditional and disentangled unitary coupled-cluster ansätze}

In UCC, the reference state is an easily prepared single determinant $\left|\Phi_{0}\right\rangle=\left|\psi_{1} \psi_{2} \cdots\right\rangle$ specified by the occupied spin orbitals $\left\{\psi_{i}\right\}$. A UCC unitary is parameterized using a pool of anti-Hermitian operators $\mathcal{P}=$ $\left\{\hat{\kappa}_{\mu}: \mu=1, \ldots, N_{\mathrm{op}}^{\mathrm{pool}}\right\}$. A generic anti-Hermitian operator $\hat{\kappa}_{\mu}=\hat{\tau}_{\mu}-\hat{\tau}_{\mu}^{\dagger}$ is defined in terms of the particlehole excitation operators $\hat{\tau}_{\mu} \equiv \hat{\tau}_{i j \cdots}^{a b \cdots}=\hat{a}_{a}^{\dagger} \hat{a}_{b}^{\dagger} \cdots \hat{a}_{j} \hat{a}_{i}$. Note that we have reinterpreted $\mu$ as the multi-index $\mu \equiv$ $[(i, j, \ldots),(a, b, \ldots)]$ of unique excitations from hole (occupied) $\left(\psi_{i} \psi_{j} \cdots\right)$ to particle (unoccupied) $\left(\psi_{a} \psi_{b} \cdots\right)$ spin orbitals. Using this parameterization, when a cluster operator $\hat{\kappa}_{\mu}$ acts on the reference, it generates elements of the many-body basis (excited determinants) of the form

$$
\left|\Phi_{\mu}\right\rangle=\hat{\kappa}_{\mu}\left|\Phi_{0}\right\rangle=\left|\Phi_{i j \cdots}^{a b \cdots}\right\rangle,
$$

and since in the case of a UCC (or dUCC) ansatz there is a one-to-one correspondence between operators and determinants, we may label them with the same index. Note that this operator basis satisfies the orthonormality condition $\left\langle\Phi_{0}\left|\hat{\kappa}_{\mu}^{\dagger} \hat{\kappa}_{\nu}\right| \Phi_{0}\right\rangle=\left\langle\Phi_{\mu} \mid \Phi_{\nu}\right\rangle=\delta_{\mu \nu}$. 
In traditional UCC [42-46], the wave function is generated by an exponential operator

$$
\hat{U}(\mathbf{t})=e^{\hat{\sigma}}=e^{\sum_{\mu} t_{\mu} \hat{\kappa}_{\mu}},
$$

assuming that the cluster amplitudes $t_{\mu}$ are real. In principle, it is possible to construct a circuit that exactly implements the action of the UCC operator defined in Eq. (7), but in practice it is common to use a unitary with a simpler, and shallower, circuit. This is frequently accomplished using a disentangled (factorized) form of the UCC ansatz

$$
\hat{U}(\mathbf{t})=\prod_{\mu} e^{t_{\mu} \hat{\kappa}_{\mu}} .
$$

Because the operators $\hat{\kappa}_{\mu}$ do not commute, an ansatz of the disentangled form is uniquely defined by an ordered set (or subset) of operators $\mathcal{A}=\left(\hat{\kappa}_{\mu_{i}}: i=1, \ldots, N_{\mathrm{op}}\right)$ built from the pool $\mathcal{P}$. The operators in $\mathcal{A}$ are then used to form an ordered product of exponential unitaries

$$
\hat{U}(\mathbf{t})=e^{t_{\mu_{1}} \hat{\kappa}_{\mu_{1}}} e^{t_{\mu_{2}} \hat{\kappa}_{\mu_{2}}} \ldots e^{t_{\mu_{N_{\mathrm{op}}}} \hat{\kappa}_{N_{\mathrm{op}}}},
$$

where $t_{\mu_{i}}$ is the amplitude corresponding to the operator $\hat{\kappa}_{\mu_{i}}$.

The disentangled UCC ansatz may be viewed as a firstorder Suzuki-Trotter approximation to UCC; however, it was recently shown [69] that dUCC is substantially different from conventional UCC [42-46]. An arbitrary quantum state can always be represented in the form of Eq. (9) using particle-hole excitation and de-excitation operators [69]. However, only certain operator orderings $\mathcal{A}$ of a complete operator pool (containing up to $N$-body particle-hole excitations) can represent any quantum state. Nevertheless, orderings that do not satisfy this condition can exactly represent states that are close to the reference. In this work we assume that all operators appear at most once in $\mathcal{A}$, but the more general case in which $\mathcal{A}$ contains repetitions has also been considered in other contexts [36,71].

For PQE formulated using a UCC or dUCC trial state, it is possible to show (see Appendix A) that, for an exact ansatz, the residual condition [Eq. (4)] and the VQE energy stationarity condition $\left(\partial E_{\mathrm{VQE}} / \partial t_{\mu}=0\right)$ are equivalent. However, for an approximate ansatz, we find that the gradient of the PQE energy contains a contribution due to the nonzero residual elements corresponding to the subspace $S$ :

$$
\frac{\partial E_{\mathrm{PQE}}(\mathbf{t})}{\partial t_{\mu}}=2 \operatorname{Re} \sum_{\Phi_{\nu} \in S} r_{\nu}^{*}\left\langle\Phi_{\nu}\left|\hat{U}^{\dagger}(\mathbf{t}) \frac{\partial \hat{U}(\mathbf{t})}{\partial t_{\mu}}\right| \Phi_{0}\right\rangle .
$$

Here $\mathbf{t}$ is a solution of the PQE equations in the projection space $R$. Suppose that $R$ is chosen to be the space of single and double excitations and that $S$ is its complement. Then this result shows that even if $r_{\mu}=0$ for all singles and doubles, the gradient of $E_{\mathrm{PQE}}$ with respect to singles and doubles may not be zero because residuals $r_{v}$ corresponding to triple and higher excitations and the term $\left\langle\Phi_{0}\left|\hat{U}^{\dagger}(\mathbf{t}) \partial \hat{U}(\mathbf{t}) / \partial t_{\mu}\right| \Phi_{\nu}\right\rangle$ are generally not null. Therefore, $\mathrm{PQE}$ and $\mathrm{VQE}$ energies obtained from approximate ansätze will be different.

We note that the combination of PQE and dUCC produces energies that are additive for noninteracting fragments (size consistent) when using a localized basis. This property follows from the fact that dUCC excitation operators for noninteracting fragments act only on orbitals localized on each fragment, and, therefore, commute. Consequently, the dUCC wave function is multiplicatively separable (as long as the order of the operators within a fragment is preserved). We have verified numerically that dUCC with single and double trial states optimized with PQE energy are size consistent by performing calculations on $\mathrm{H}_{4}+\mathrm{H}_{2}$ separated at a $1000 \AA$ and verified to within numerical convergence $\left(10^{-10} E_{h}\right)$ that the energy is additive in the fragments.

\section{Numerical solution of the dUCC PQE amplitude equation}

To realize the PQE scheme on a quantum computer, the reference state, the Hamiltonian, and the unitary must be represented in a qubit basis via a fermionic mapping. After such a transformation, the Hamiltonian is a sum of the form $\hat{H}=\sum_{\ell} h_{\ell} \hat{O}_{\ell}$, where $h_{\ell}$ is an electron integral multiplied by a coefficient and $\hat{O}_{\ell}=\prod_{i} \hat{\sigma}_{j_{\ell_{i}}}^{q \ell_{i}}$ is a unique product of $\hat{\sigma}_{x}, \hat{\sigma}_{y}$, or $\hat{\sigma}_{z}$ Pauli operators acting on qubits $q_{\ell_{i}}$. Similarly, each term in the unitary $\exp \left(t_{\mu} \hat{\kappa}_{\mu}\right)$ may be implemented using a combination of one- and two-qubit operators following standard approaches [33-35].

To solve the PQE equations, we measure the residuals corresponding to the operators contained in $\mathcal{A}$ on a quantum computer and update the parameter vector using a simple quasi-Newton iteration approach

$$
t_{\mu}^{(n+1)}=t_{\mu}^{(n)}+\frac{r_{\mu}^{(n)}}{\Delta_{\mu}}
$$

where the superscript " $(n)$ " indicates the amplitude at iteration $n$. The quantities $\Delta_{\mu}$ are standard MøllerPlesset denominators $\Delta_{\mu} \equiv \Delta_{i j \cdots}^{a b \cdots}=\epsilon_{i}+\epsilon_{j}+\cdots-\epsilon_{a}-$ $\epsilon_{b} \cdots$, where the $\epsilon_{i}$ are Hartree-Fock orbital energies. This update equation is derived in Appendix B using Newton's method and taking the leading contributions to the Jacobian to be the diagonal elements of the Fock operator [72]. It is further assumed that the amplitudes are small, so that the Jacobian can be approximated by terms linear in the operators $\hat{\kappa}_{\mu}$ and issues with noncommuting operators are avoided. Therefore, convergence of this 
quasi-Newton scheme is not mathematically guaranteed if one or more amplitudes are large. We found it useful to improve numerical stability and speed up convergence of the PQE equations, to combine amplitude updates via Eq. (11) with the direct inversion of the iterative subspace (DIIS) convergence accelerator algorithm [73,74].

It is important to note that the current formulation of $\mathrm{PQE}$ is compatible with any ansatz such that the metric matrix

$$
S_{\mu_{i} \mu_{j}}=\left\langle\Phi_{0}\left|\hat{\kappa}_{\mu_{i}}^{\dagger} \hat{\kappa}_{\mu_{j}}\right| \Phi_{0}\right\rangle \quad \text { for all } \hat{\kappa}_{\mu_{i}}, \hat{\kappa}_{\mu_{j}} \in \mathcal{A}
$$

is the identity. In more general cases (e.g., when $S_{\mu_{i} \mu_{j}}$ is nondiagonal or singular), the PQE formalism requires a generalization of the amplitude update equations or the use of residual norm minimization instead of Eq. (4). These variants of $\mathrm{PQE}$ would allow one to consider ansätze that contain repeated operators in $\mathcal{A}$, employ general many-body operators, or a basis of qubit operators. These extensions go beyond the scope of this work and will be considered in future studies.

There are two advantages to the combination of the PQE and dUCC described above. Firstly, as we show in the following subsection, one element of the residual vector $\left(r_{\mu}\right)$ can be evaluated with essentially the same resources required to measure the energy in VQE. Secondly, the magnitude of the residuals provides an indication of the importance of an operator $\hat{\kappa}_{\mu}$, which in turn may be used to define a selection procedure to form the sequence of unitaries that enter in $\hat{U}(\mathbf{t})$. The next two subsections describe these two points in detail.

\section{Efficient measurement of the residual elements}

For a trial state built from the ordered pool $\mathcal{A}$, the number of the residual elements that must be evaluated to solve the PQE equations is equal to the size of the pool $|\mathcal{A}|$. The PQE residuals can be expressed as the off-diagonal matrix elements of the operator $\bar{H}=\hat{U}^{\dagger}(\mathbf{t}) \hat{H} \hat{U}(\mathbf{t})$ as $r_{\mu}=\left\langle\Phi_{\mu}|\bar{H}| \Phi_{0}\right\rangle$ (we use this notation throughout the paper, but we note that we never explicitly form the operator $\bar{H}$ on a classical computer). Then, in principle, the residuals can be measured on a quantum computer using a variant of the Hadamard test [75], but we have found an ancilla-free procedure in which these matrix elements are computed by measuring diagonal quantities. Acting on the reference with the operator $e^{\theta \hat{\kappa}_{\mu}}$ yields the state

$$
\left|\Omega_{\mu}(\theta)\right\rangle=e^{\theta \hat{\kappa}_{\mu}}\left|\Phi_{0}\right\rangle=\cos (\theta)\left|\Phi_{0}\right\rangle+\sin (\theta)\left|\Phi_{\mu}\right\rangle,
$$

noting that the above expression is valid because $\hat{\kappa}_{\mu}\left|\Phi_{0}\right\rangle$ $=\left|\Phi_{\mu}\right\rangle$ and $\hat{\kappa}_{\mu}^{2}\left|\Phi_{0}\right\rangle=-\left|\Phi_{0}\right\rangle$ (see also Refs. [47,48]). Taking the expectation value of the similarity transformed Hamiltonian with respect to $\Omega_{\mu}(\theta)$ using $\theta=\pi / 4$, and noting that the wave function is real, leads to the following equation for the residual elements:

$$
r_{\mu}=\left\langle\Omega_{\mu}(\pi / 4)|\bar{H}| \Omega_{\mu}(\pi / 4)\right\rangle-\frac{1}{2} E_{\mu}-\frac{1}{2} E_{0} .
$$

Here $E_{0}=\left\langle\Phi_{0}|\bar{H}| \Phi_{0}\right\rangle$ and $E_{\mu}=\left\langle\Phi_{\mu}|\bar{H}| \Phi_{\mu}\right\rangle$. All of these quantities are expectation values of $\bar{H}$ with respect to reference states that are easily generated with short quantum circuits. The evaluation of the exact residual via Eq. (14) has a cost similar to the evaluation of exact gradients in VQE via the shift rule $[63,64]$.

\section{E. Efficient operator selection}

In this section, we generalize the PQE method to utilize a flexible dUCC ansatz built iteratively using a full operator pool. As shown in the case of VQE [36,65], significantly more compact and flexible approximations may be achieved if the operators that define the unitary are selected according to an importance criterion. To formulate a selected version of the PQE approach, we propose to combine information about the residual with a cumulative importance criterion. Since the residuals $r_{\mu}$ are zero for an eigenstate, we propose to estimate the importance of the operators $\hat{\kappa}_{\mu}$ using the magnitude of the residual $\left(\left|r_{\mu}\right|\right)$. However, instead of evaluating the importance of all the operators in the pool via operator averaging (like in gradient-based selection schemes [36,65]), we propose to sample a quantum state whose probability amplitudes encode the importance of all operators up to rank $N$.

Suppose that we have determined a unitary $\hat{U}$ that satisfies the residual condition $r_{\mu}=0$ for all $\hat{\kappa}_{\mu}$ in the current ordered set $\mathcal{A}$. In our approach we prepare a (normalized) quantum state of the form $|\tilde{r}\rangle=\tilde{r}_{0}\left|\Phi_{0}\right\rangle+\sum_{\mu} \tilde{r}_{\mu}\left|\Phi_{\mu}\right\rangle$, where the quantities $\tilde{r}_{\mu}$ are approximately proportional to the residuals $r_{\mu}$. When $|\tilde{r}\rangle$ is represented in a qubit basis, there is a one-to-one mapping between elements of the computational basis and the states $\Phi_{\mu}$. Therefore, a measurement of the state $|\tilde{r}\rangle$ in the qubit basis will yield one of the states $\Phi_{\mu}$ with probability $P_{\mu}=\left|\tilde{r}_{\mu}\right|^{2}$. Repeated measurement of the state $|\tilde{r}\rangle$ provides a way to approximately determine the elements of the residual $r_{\mu}$ with the largest magnitude, and the corresponding operators $\hat{\kappa}_{\mu}$ that should be included in the unitary. When this strategy is combined with an efficient way to prepare the state $|\tilde{r}\rangle$, it is much more cost effective than evaluating all the elements of $r_{\mu}$ not included in $\mathcal{A}$ via operator averaging [Eq. (14)].

Construction of the state $|\tilde{r}\rangle$ would require one to apply the Hamiltonian, which is not a unitary operator. Therefore, we evaluate $\tilde{r}$ using the unitary operator $e^{-i \Delta t \hat{H}}=$ $1-i \Delta t \hat{H}+\mathcal{O}\left(\Delta t^{2}\right)$ instead of $\hat{H}$. By choosing a small time step, we can ensure that the nonlinear terms and errors due to the approximate implementation of $e^{-i \Delta t \hat{H}}$ (e.g., via Trotterization) do not affect the residual to leading order in 
$\Delta t$. The residual state can then be defined as

$$
\begin{aligned}
|\tilde{r}\rangle & =\hat{U}^{\dagger} e^{i \Delta t \hat{H}} \hat{U}\left|\Phi_{0}\right\rangle \\
& =\left(1+i \Delta t \hat{U}^{\dagger} \hat{H} \hat{U}\right)\left|\Phi_{0}\right\rangle+\mathcal{O}\left(\Delta t^{2}\right) .
\end{aligned}
$$

The time-evolution operator may be approximated via Trotterization $[25,26]$ in combination with low-rank representations of the Hamiltonian [76]. With a sufficiently large number of measurements $M$ of the state $|\tilde{r}\rangle$, we may approximate the values of the (normalized) squared residuals as

$$
\left|\tilde{r}_{\mu}\right|^{2} \approx \frac{N_{\mu}}{M},
$$

where $N_{\mu}$ is the number of times the state $\left|\Phi_{\mu}\right\rangle$ is measured. Encoding the residual in a single quantum state allows us to efficiently sample the entire operator pool without the need to generate and store individual elements of the residual vector in memory. This distinctive feature makes it possible to employ this selection procedure with an operator pool that includes particle-hole operators of arbitrary order.

To select important operators, we adopt a cumulative threshold approach that allows us to add a batch of operators at a time. Our goal is to iteratively construct a unitary that contains the fewest operators. This is realized with a selection procedure that adds the operators with the largest value of $\tilde{r}_{\mu}$ to $\mathcal{A}$ (as motivated by the Gershgorin circle theorem bounds discussed in Sec. II A), and excludes all other operators in such a way that sum of their residuals squared is less than a threshold $\Omega^{2}$. Specifically, we enforce that

$$
\sum_{\hat{\kappa}_{\mu} \notin \mathcal{A}}^{\text {excluded }}\left|r_{\mu}\right|^{2} \approx \sum_{\hat{\kappa}_{\mu} \notin \mathcal{A}}^{\text {excluded }} \frac{\left|\tilde{r}_{\mu}\right|^{2}}{\Delta t^{2}} \leq \Omega^{2},
$$

where we have used the fact that $\left|\tilde{r}_{\mu}\right| \approx \Delta t\left|r_{\mu}\right|$. In practice, we sort the operators in ascending order according to $\left|\tilde{r}_{\mu}\right|^{2}$, and starting from the first element, we discard operators until Eq. (17) is satisfied. The remaining operators are appended to the end of $\mathcal{A}$ in order of decreasing $\left|\tilde{r}_{\mu}\right|^{2}$. The resulting operator ordering is consistent with the following renormalization transformation of the Hamiltonian that begins with the largest many-body rotation and gradually continues with smaller ones:

$$
\begin{aligned}
& \hat{H} \rightarrow e^{-t_{\mu_{1}} \hat{\kappa}_{\mu_{1}}} \hat{H} e^{t_{\mu_{1}}}=\bar{H}_{1} \\
& \rightarrow e^{-t_{\mu_{2}} \hat{\kappa}_{\mu_{2}}} \bar{H}_{1} e^{t_{\mu_{2}} \hat{\kappa}_{\mu_{2}}}=\bar{H}_{2} \\
& \rightarrow \cdots \text {. }
\end{aligned}
$$

Note that this ordering is the reverse of that used in ADAPT VQE and iQCC, where operators with the largest gradient are applied first to the reference. In applications of this selection scheme, we found that our operator ordering is more numerically robust and accurate than its reverse. This selection procedure is easily integrated in the PQE algorithm by performing a series of computations with increasingly larger ordered sets. When no new operators are added to $\mathcal{A}$, the computation is considered converged, and the final operator set satisfies Eq. (17). The details of the selected PQE algorithm are discussed in Sec. II F.

Throughout this work, we ignore errors that arise from finite measurement of the approximate residual $|\tilde{r}\rangle$. However, since operator selection only requires an approximate determination of the $\left|\tilde{r}_{\mu}\right|^{2}$ values, it is not necessary to perform a large number of measurements. Indeed, in Appendix E, we discuss a simple strategy based on sampling $|\tilde{r}\rangle$ a fixed number of times that performs as well as the exact scheme.

\section{F. Outline of the selected PQE algorithm}

The combination of the PQE approach with the selection procedure described in Sec. IIE leads to a very efficient flexible ansatz quantum algorithm, which we refer to as selected PQE. The selected PQE algorithm requires the simultaneous solution of the residual conditions and the selection of important excitation operators. To realize this scheme, we alternate microiterations to converge the residual equations (for the current ordered operator set) with macroiterations that perform importance selection of new operators. The selected PQE procedure is illustrated in Fig. 2 and consists of the following steps.

1. Initialization. The user provides the occupation numbers that define the reference state $\Phi_{0}$. Start at macroiteration number $k=0$ with an empty operator $\operatorname{set}\left(\mathcal{A}^{(0)}=\{\}\right)$.

2. Importance selection. At macroiteration $k$, perform $M$ measurements in the computational basis of the state $\left|\tilde{r}^{(k)}\right\rangle=\hat{U}^{(k) \dagger} e^{i \Delta t \hat{H}} \hat{U}^{(k)}\left|\Phi_{0}\right\rangle$. The number of times the state $\Phi_{\mu}$ is measured is accumulated in the variable $N_{\mu}$. These numbers are used to estimate the square residuals via Eq. (16), which are in turn used to select important excitations not included in the current pool $\mathcal{A}_{k}$. The current operator set $\mathcal{A}_{k}$ and all the new selected operators are included in the new set $\mathcal{A}_{k+1}$. When forming this new ordered set, we append the new operators - sorted in decreasing value of the approximate squared residual - to $\mathcal{A}_{k}$. If the sum in Eq. (17) over all approximate residuals is less than the threshold $\Omega^{2}$, such that no new operators are added, then return the final energy.

3. Solution of the $P Q E$ equations. Using the new set $\mathcal{A}_{k+1}$, solve the PQE equations via quasi-Newton microiterations. These microiterations alternate the evaluation of the residuals [Eq. (4)] and the 


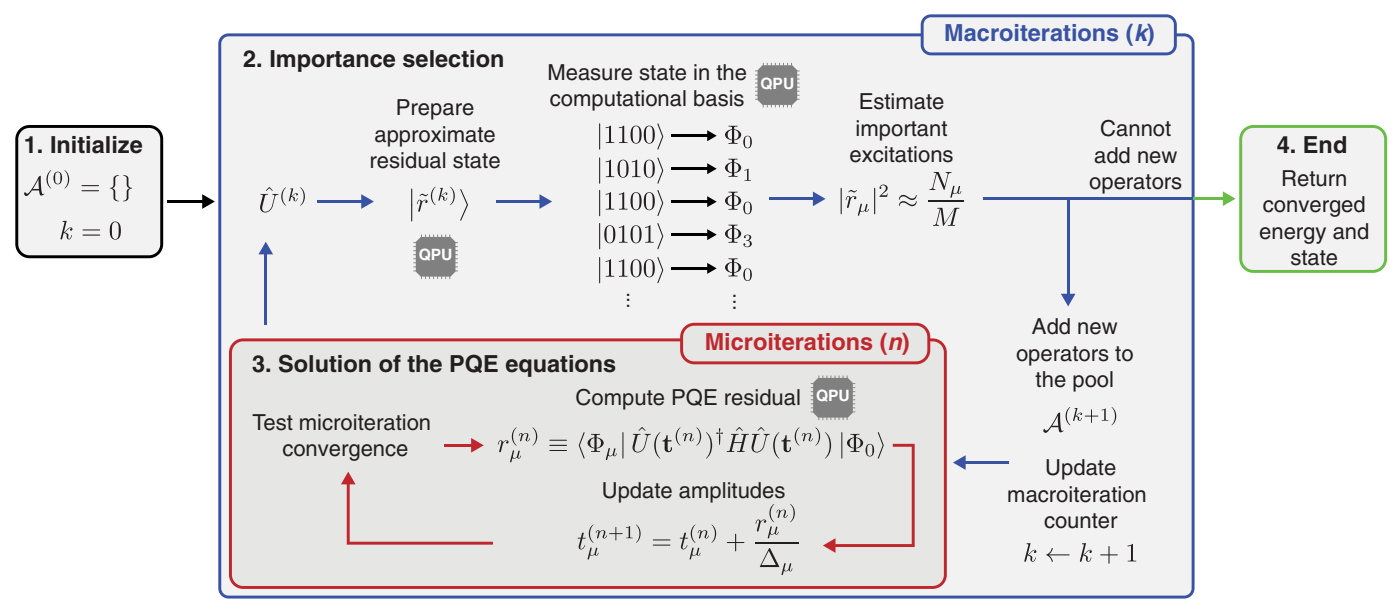

FIG. 2. Outline of the selected PQE algorithm. Steps labeled "QPU" indicate parts of the algorithm that run on a quantum processing unit.

amplitude update [Eq. (11)]. The microiterations are considered converged when the norm of the residual vector $\|\mathbf{r}\|$ is less than a user specified threshold $\omega_{r}$. Once converged, this step produces a new set of amplitudes $\left[\mathbf{t}^{(k+1)}\right]$ and the corresponding unitary $\left[\hat{U}^{(k+1)}\right]$, as well as the updated energy $\left[E^{(k+1)}\right]$. Increase the macroiteration number by one $(k \leftarrow$ $k+1)$ and go to step 2 .

All VQE and PQE methodologies are implemented in a development branch of the open source package QFORTE, and utilize its state-vector simulator. All VQE calculations use a microiteration convergence threshold $\omega_{g}=10^{-5} E_{h}$ for the gradient norm $\|\mathbf{g}\|$ and all PQE calculations use a microiteration threshold $\omega_{r}=10^{-5} E_{h}$ for the residual norm $\|\mathbf{r}\|$. Note that, when the $\hat{\kappa}_{\mu}$ operators are mapped to a qubit basis, they can be expressed as a sum of Pauli operator strings that commute [77], and, therefore, a single operator $e^{t_{\mu} \hat{\kappa}_{\mu}}$ can be implemented exactly as a product of exponentials without invoking the Trotter approximation.

\section{RESULTS AND DISCUSSION}

\section{A. Comparison of PQE and VQE with a disentangled UCC ansatz}

Our initial goal is to compare the performance of $\mathrm{PQE}$ and VQE using a unitary coupled-cluster trial state truncated at a given particle-hole excitation level. We test these two methods on a family of linear hydrogen chains (with identical nearest-neighbor distance) ranging from four to ten atoms, both near their equilibrium geometries $\left(r_{\mathrm{H}-\mathrm{H}}=0.75 \AA\right)$ and stretched geometries $\left(r_{\mathrm{H}-\mathrm{H}}=1.5 \AA\right)$. Hydrogen models such as these have been studied experimentally with VQE [78] and have recently been used as a benchmark for both quantum [36,55] and classical [79-81] algorithms.
Figure 3 shows the energy convergence of $\mathrm{PQE}$ and VQE using a disentangled UCC ansatz with singles and doubles (dUCCSD). All calculations employed restricted Hartree-Fock (RHF) orbitals from the quantum chemistry package PSI4 [82], and Pauli-operator Hamiltonians obtained via the Jordan-Wigner transformation implemented in QFORTE [83]. To achieve optimal performance for both VQE and PQE, we employ the Broyden-FletcherGoldfarb-Shannon (BFGS) algorithm [84-87] (as implemented in the SCIPY [88] scientific computing library) with analytical gradients for VQE, and DIIS [73,74] to accelerate amplitude convergence of PQE. These computations use the same operator ordering for both approaches, with all amplitudes initialized to zero. The ordering of the operators $e^{t_{\mu_{i}} \hat{\kappa}_{\mu_{i}}}$ entering Eq. (9) is defined by the binary representation of the corresponding determinants $\left|\Phi_{\mu_{i}}\right\rangle=\hat{\tau}_{\mu_{i}}\left|\Phi_{0}\right\rangle$ in the occupation number representation. Because the disentangled UCCSD state cannot exactly parameterize an eigenstate of the Hamiltonian, the numerically converged PQE and VQE energies are not identical. Nevertheless, for all the cases we examined, the converged PQE and VQE energies differ by less than $10^{-6} E_{h}$.

Near the equilibrium geometry, we find that the PQE energy converges significantly faster than the VQE energy with the number of residual versus gradient evaluations, respectively. For example, to converge the nearequilibrium $\mathrm{H}_{10}$ energy to $10^{-6} E_{h}$, $\mathrm{PQE}$ requires only seven residual evaluations, while $\mathrm{VQE}$ requires approximately 23 gradient evaluations. In the case of VQE, we also observe that the number of required gradient evaluations grows with system size, with $\mathrm{H}_{10}$ taking twice as many gradient vector evaluations than $\mathrm{H}_{4}$ to converge. On the contrary, PQE computations converge with similar speed for all equilibrium hydrogen systems. Plots of the energy change versus the norm of the residual or gradient vector show similar trends and are reported in Appendix C. 

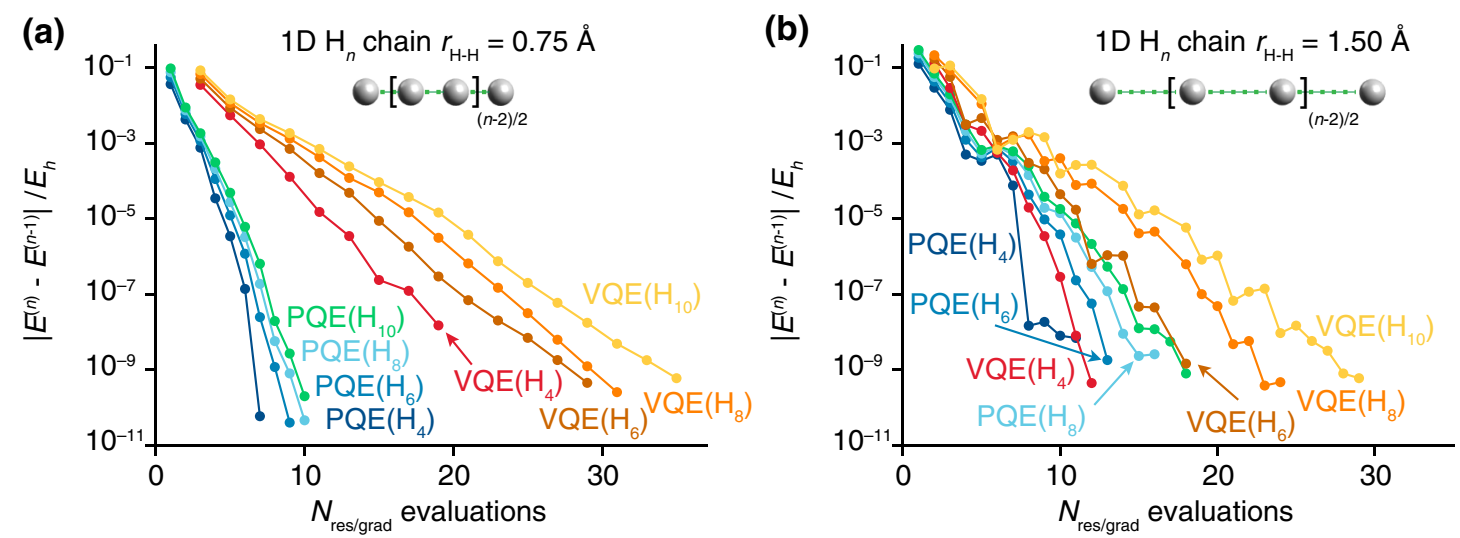

FIG. 3. dUCCSD energy convergence for linear $\mathrm{H}_{4}-\mathrm{H}_{10}$ chains in a STO-6G basis at (a) $r_{\mathrm{H}-\mathrm{H}}=0.75 \AA$, and (b) $r_{\mathrm{H}-\mathrm{H}}=1.50 \AA$. Here $\left|E^{(n)}-E^{(n-1)}\right|$ is the absolute value of the energy change between subsequent iterations. Both plots compare PQE versus VQE convergence with respect to the number of residual (for PQE) or gradient (for VQE) evaluations $\left(N_{\text {res/grad }}\right)$.

At the stretched geometry, strong correlation effects cause the disentangled UCCSD trial state to perform more poorly, with both $\mathrm{PQE}$ and VQE, yielding energy errors that range from $1.39 \mathrm{~m} E_{h}$ (for $\mathrm{H}_{4}$ ) to $13.59 \mathrm{~m} E_{h}$ (for $\mathrm{H}_{10}$ ). We see that PQE converges slightly more slowly in the stronger correlation regime, with stretched $\mathrm{H}_{10}$ requiring 13 residual evaluations (instead of seven) to converge the energy to $10^{-6} E_{h}$. However, PQE always converges faster than $\mathrm{VQE}$, with the latter requiring 19 gradient-vector evaluations to converge stretched $\mathrm{H}_{10}$ to the same accuracy level. We also find similar trends in PQE and VQE convergence for $\mathrm{BeH}_{2}$, for which convergence data can be found in Appendix C.

In summary, this initial set of results suggests that, for a given trial state, optimization via PQE is faster than VQE and less dependent on the number of parameters to optimize. We expect this to be the case also for VQE based on numerical gradients or gradient-free optimization methods, since these two variants are known to be slower compared to the BFGS approach adopted here [77].

\section{B. Effect of stochastic errors on the convergence of PQE and VQE}

The results presented in the previous section assume error-free quantum gates and arbitrarily precise measurements. In practice, calculations performed on NISQ hardware are affected by decoherence errors, poor gate fidelity, readout errors, and loss of precision due to insufficient measurements. These sources of error will lead to incorrect gradients and residuals that are then passed to a classical optimizer. Therefore, it is interesting to compare the resilience of PQE and VQE procedures when the residuals and gradients are affected by stochastic errors.

To model the presence of errors, we modify the PQE procedure by adding to the residual vector a stochastic error sampled from a Gaussian distribution with standard deviation $\sigma\left[\mathcal{N}\left(0, \sigma^{2}\right)\right]$,

$$
r_{\mu}^{\text {measured }}=r_{\mu}+\mathcal{N}\left(0, \sigma^{2}\right) .
$$

For VQE, we similarly add stochastic noise to the exact energy gradients. Using Eq. (19) as a noise model mainly emulates errors that arise from finite measurement. Because the inexact residuals $r_{\mu}^{\text {measured }}$ are used to update the cluster amplitudes via Eq. (11), this noise model also gives rise to control errors, or errors that refer to the difference between the unitaries for noiseless updated amplitudes $\hat{U}(\mathbf{t})$ and noisy updated amplitudes $\hat{U}(\mathbf{t}+\Delta \mathbf{t})$. Control errors due to finite measurement have been modeled this way in previous studies [77] and will always propagate through optimization on physical hardware. We note, however, that using Eq. (19) exclusively as a noise model is insufficient to capture more nuanced or device-specific errors such as decoherence. We tested the performance of PQE and VQE under noise by performing a batch of 50 computations on the linear $\mathrm{H}_{4}$ molecule with nearestneighbor distance set to $1.0 \AA$. We use the disentangled UCC ansatz with up to quadruple excitations, which spans the full operator set for this system.

Figure 4 shows a comparison of PQE and VQE optimized with various levels of noise, controlled by the magnitude of $\sigma$ in Eq. (19). We find that, for all values of $\sigma>0$, the energy convergence of $\mathrm{PQE}$ is essentially identical to that of noiseless PQE until some point, after which the energy error hovers around a finite value. Similar behavior can be seen for convergence of the residual vector. We find that $\mathrm{VQE}$ has similar characteristics to $\mathrm{PQE}$ in the presence of noise, but that it is able to achieve slightly more accurate energies for a given $\sigma$ value. With the same noise level, however, VQE generally requires 2-3 times the number of gradient evaluations as the number of residual evaluations required by PQE. An important aspect of this comparison is that, for a given $\sigma$, both the 


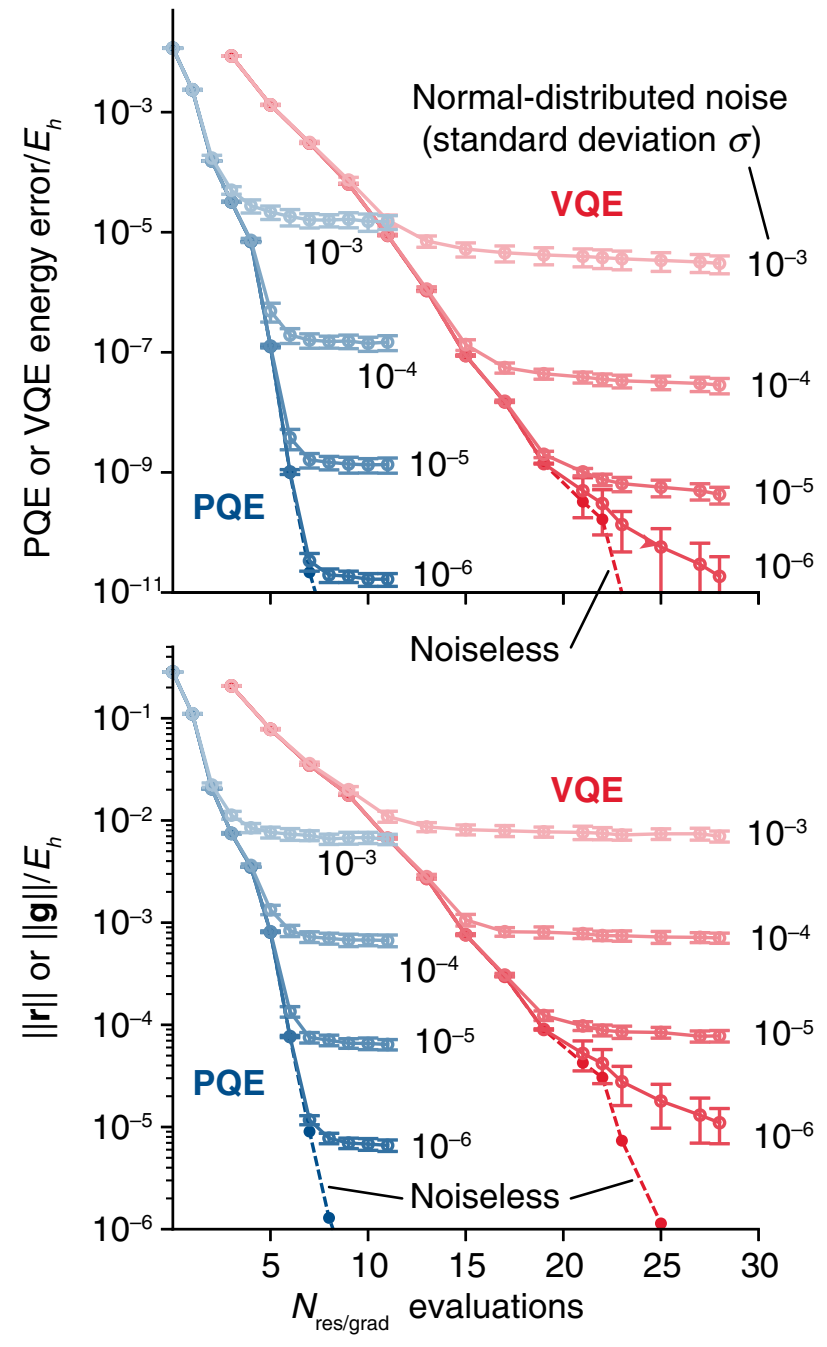

FIG. 4. Energy and residual norm $(\|\mathbf{r}\|)$ or gradient norm $(\|\mathbf{g}\|)$ convergence of dUCCSDTQ wave functions optimized with PQE and VQE with various amounts of stochastic noise added to the residuals and gradients. The energy error is relative to FCI. Here $\sigma$ controls the degree of noise and is the standard deviation of the normal distribution, centered at the exact residual or gradient value, from which all residuals and gradients used in the calculations are randomly sampled [see Eq. (19)]. Values at each PQE or VQE iteration are averages over 50 runs on $\mathrm{H}_{4}$ at $r_{\mathrm{H}-\mathrm{H}}=1.0 \AA$ A. Error bars denote one standard deviation.

residual and gradient vectors yield comparable asymptotic errors in PQE and VQE, respectively. Conversely, PQE and $\mathrm{VQE}$ computations of the comparable energy accuracy require similar precision in the measurement of the residual and gradient vectors. In Appendix D we use this result to estimate the relative cost of PQE and VQE via a formal analysis.

\section{Selected PQE based on a full dUCC operator pool}

Here we compare the results of SPQE with an arbitrary order particle-hole operator pool, and ADAPT VQE, which unless otherwise noted, uses a generalized singles and doubles pool (operators of the form $\hat{a}_{p}^{\dagger} \hat{a}_{q}$ and $\hat{a}_{p}^{\dagger} \hat{a}_{q}^{\dagger} \hat{a}_{s} \hat{a}_{r}$, where the indices $p, q, r, s$ run over all spin orbitals). To test these methods, we compute the energy as a function of bond distances for: (1) the symmetric dissociation of the linear $\mathrm{BeH}_{2}$ molecule and (2) the symmetric dissociation of a chain of six hydrogen atoms. In both cases, there is a build up of strong correlation effects as the bond length increases. For each system, we report two sets of results for SPQE using the cumulative thresholds $\Omega=10^{-1}$ and $10^{-2} E_{h}$, and two sets of ADAPT VQE results with gradient thresholds $10^{-1}$ and $10^{-3} E_{h}\left(\epsilon_{1}\right.$ and $\epsilon_{3}$ in the original notation used by the authors). Since we later compare these methods to classical approaches formulated in a determinant basis, we do not employ a pool of spin-adapted operators.

The dissociation curve of $\mathrm{BeH}_{2}$ shown in Fig. 5(a) demonstrates that both SPQE and ADAPT VQE are able to achieve significantly smaller energy errors than dUCCSD, while using only 10-20 more parameters. Although the two approaches employ different selection schemes, the $\operatorname{ADAPT~} \operatorname{VQE}\left(\epsilon_{3}\right)$ and $\operatorname{SPQE}\left(\Omega=10^{-2}\right)$ approaches produce compact trial states with a similar number of classical parameters and comparable errors. The same trends are seen in the symmetric dissociation curve of $\mathrm{H}_{6}$, which is shown in Fig. 5(b). However, for this system, we find that achieving sub-m $E_{h}$ accuracy — particularly with the onset of strong correlation at $r_{\mathrm{H}-\mathrm{H}}$ values greater than 1.5 $\AA$-requires a number of parameters that approaches the size of the full Hilbert space (200) for both ADAPT VQE and SPQE. The need to saturate Hilbert space to accurately describe $\mathrm{H}_{6}$ is likely due to how small this example is, and it speaks more to the ability of the trial states to produce compact representations rather than the performance of these algorithms in optimizing such ansätze.

The most noticeable difference between SPQE and ADAPT VQE can be seen in the bottom panels of Figs. 5(a) and 5(b): for trial states with a comparable number of parameters, SPQE requires significantly fewer residual element evaluations than gradient element evaluations in ADAPT VQE. For example, at a Be-H bond distance of $1.65 \AA$, both methods produce very similar energy errors using almost the same number of parameters, but ADAPT $\operatorname{VQE}\left(\epsilon_{3}\right)$ requires the evaluation of 35155 elements of the gradient, whereas $\operatorname{SPQE}\left(\Omega=10^{-2}\right)$ requires only 1220 elements of the residual. Importantly, we note that the bottom panels of Fig. 5 exclusively count the number of elements of the gradient or residual required by the optimization, and do not include the additional measurements required for operator selection.

Since ADAPT VQE and SPQE select new operators from their pools using different importance criteria, it is not possible to perform a direct comparison of their performance using fixed thresholds. To facilitate this comparison, in Table I we report SPQE results using two 

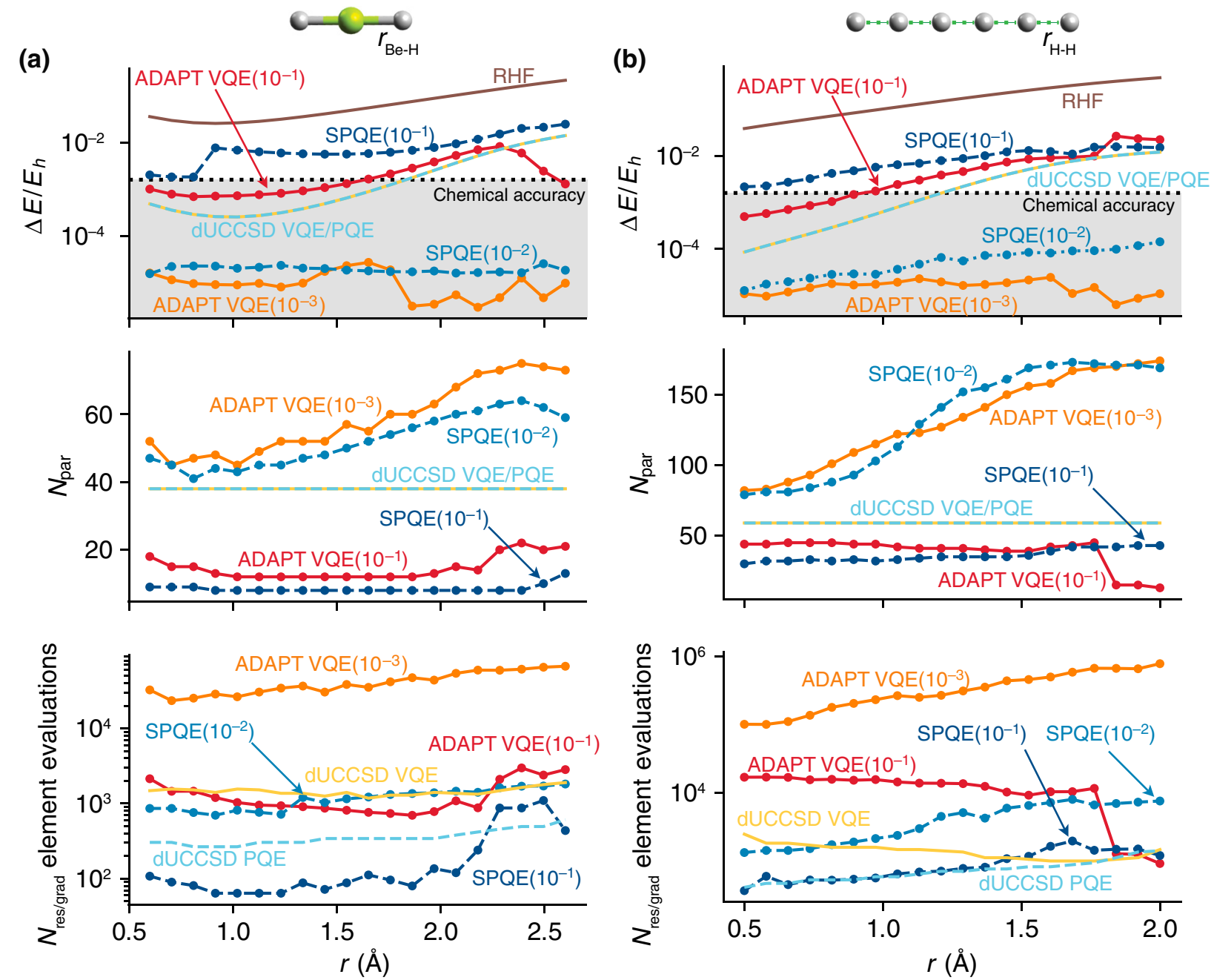

FIG. 5. Ground-state potential energy curve for the symmetric dissociation of (a) $\mathrm{BeH}_{2}$ and (b) $\mathrm{H}_{6}$ computed using a minimal (STO6G) basis. The energy error relative to FCI (top), the number of classical parameters used (middle), and the number of individual elements of the gradient (for VQE) or residual (for PQE) evaluated (bottom) are given as a function of the Be-H and $\mathrm{H}-\mathrm{H}$ bond lengths. Here, ADAPT VQE uses a generalized singles and doubles operator pool and is optimized with the BFGS algorithm, and gradient convergence thresholds $10^{-1}\left(\epsilon_{1}\right)$ and $10^{-3}\left(\epsilon_{3}\right)$. SPQE results use macroiteration convergence thresholds $\Omega=10^{-1}$ and $10^{-2}$. The top plots also show the energy error corresponding to chemical accuracy, here defined as $1 \mathrm{kcal} / \mathrm{mol} \approx 1.59 \mathrm{~m} E_{h}$.

values of $\Omega\left(10^{-1}\right.$ and $\left.10^{-2} E_{h}\right)$ together with ADAPT $\mathrm{VQE}$ results obtained using an ansatz with the same number of parameters as SPQE. We include ADAPT VQE results obtained using both a GSD operator pool, and a particle-hole SD pool. The results in Table I obtained with $\Omega=10^{-1} E_{h}$ show SPQE and the two variants of ADAPT $\mathrm{VQE}$ to perform equally well at all bond distances. The second set of results obtained with a tighter threshold $\left(\Omega=10^{-2}\right.$ ) show similar performance of the methods at short bond lengths, with two notable exceptions. First, at $r_{\mathrm{H}-\mathrm{H}}=2.0 \AA$ ADAPT VQE GSD yields more accurate results than ADAPT VQE SD and SPQE. At this point, the SPQE ansatz contains 55 singles and doubles, and 114 operators of higher rank, yielding an error of about 0.14 $\mathrm{m} E_{h}$, while ADAPT VQE GSD is an order of magnitude more accurate. Second, also at $r_{\mathrm{H}-\mathrm{H}}=2.0 \AA$, SPQE uses only 9537 residual element evaluations, while ADAPT VQE requires 719390 (GSD) and 1312127 (SD) gradient element evaluations. The evaluation of fewer residual elements in SPQE will correspond to approximately the same savings in the total number of measurements (see Appendix D).

A final important aspect to compare between SPQE and ADAPT VQE is the number of native CNOT gates, which we consider as a proxy for circuit depth. Table I reports the number of CNOT gates for the converged trial states. These numbers overestimate the actual gate count since they ignore optimizations such as the cancelation of Jordan-Wigner strings [89], especially for three- and higher-body operators. We see that at the larger threshold 
TABLE I. Ground state of $\mathrm{H}_{6}$ computed using a minimal (STO-6G) basis with a RHF orbital convergence threshold of $10^{-10} E_{h}$. Comparison of SPQE with threshold $\Omega$ and ADAPT VQE using the same number of parameters as SPQE. ADAPT VQE results are computed for both a generalized singles and doubles (GSD) operator pool and a particle-hole singles and doubles (SD) pool. The properties reported are the energy error with respect to FCI [ $\Delta E$, in $E_{h}$ ], the number of classical parameters used [ $N_{\text {par }}$, the number of parameters corresponding to three-body or higher excitations $\left[N_{\mathrm{T}+}\right]$, the number of CNOT gates used in the unitary $\left[N_{\mathrm{CNOT}}\right]$ (not optimized), and the total number of residual or gradient element evaluations $\left[N_{\text {res }}\right.$ or $\left.N_{\text {grad }}\right]$. By $r$ we denote the H-H nearest-neighbor distance in angstroms.

\begin{tabular}{|c|c|c|c|c|c|c|c|c|c|c|c|c|c|}
\hline \multirow[b]{2}{*}{$r$} & \multicolumn{5}{|c|}{$\operatorname{SPQE}\left(\Omega=10^{-1} E_{h}\right)$} & \multicolumn{4}{|c|}{ ADAPT VQE GSD } & \multicolumn{4}{|c|}{ ADAPT VQE SD } \\
\hline & $\Delta E$ & $N_{\text {par }}$ & $N_{\mathrm{T}+}$ & $N_{\mathrm{CNOT}}$ & $N_{\text {res }}$ & $\Delta E$ & $N_{\mathrm{par}}$ & $N_{\mathrm{CNOT}}$ & $N_{\text {grad }}$ & $\Delta E$ & $N_{\mathrm{par}}$ & $N_{\mathrm{CNOT}}$ & $N_{\text {grad }}$ \\
\hline 0.50 & 0.002153 & 30 & 0 & 2400 & 339 & 0.002152 & 30 & 2400 & 8378 & 0.002152 & 30 & 2400 & 8378 \\
\hline 1.00 & 0.006050 & 32 & 0 & 2720 & 503 & 0.005872 & 32 & 2720 & 8399 & 0.005872 & 32 & 2720 & 8399 \\
\hline 1.50 & 0.012487 & 36 & 0 & 2944 & 1103 & 0.011176 & 36 & 3040 & 8046 & 0.011176 & 36 & 3040 & 8046 \\
\hline \multirow[t]{2}{*}{2.00} & 0.015066 & 43 & 8 & 20272 & 1087 & 0.011204 & 43 & 3560 & 15176 & 0.010350 & 43 & 3312 & 12592 \\
\hline & \multicolumn{5}{|c|}{$\operatorname{SPQE}\left(\Omega=10^{-2} E_{h}\right)$} & \multicolumn{4}{|c|}{ ADAPT VQE GSD } & \multicolumn{4}{|c|}{ ADAPT VQE SD } \\
\hline$r$ & $\Delta E$ & $N_{\text {par }}$ & $N_{\mathrm{T}+}$ & $N_{\mathrm{CNOT}}$ & $N_{\text {res }}$ & $\Delta E$ & $N_{\mathrm{par}}$ & $N_{\mathrm{CNOT}}$ & $N_{\text {grad }}$ & $\Delta E$ & $N_{\mathrm{par}}$ & $N_{\mathrm{CNOT}}$ & $N_{\text {grad }}$ \\
\hline 0.50 & 0.000013 & 79 & 24 & 15568 & 1127 & 0.000012 & 79 & 6608 & 87506 & 0.000085 & 79 & 4608 & 29707 \\
\hline 1.00 & 0.000031 & 105 & 46 & 33232 & 2076 & 0.000033 & 105 & 9244 & 166119 & 0.000064 & 105 & 8128 & 388182 \\
\hline 1.50 & 0.000079 & 166 & 111 & 166032 & 6074 & 0.000004 & 166 & 14528 & 566143 & 0.000028 & 166 & 12576 & 1437917 \\
\hline 2.00 & 0.000141 & 169 & 114 & 226768 & 9537 & 0.000018 & 169 & 14776 & 719390 & 0.000096 & 169 & 12944 & 1312127 \\
\hline
\end{tabular}

values $\left(\Omega=10^{-1}\right.$ for SPQE and $\epsilon_{1}=10^{-1}$ for ADAPT $)$ the numbers of CNOT gates for all three approaches are relatively similar, and are generally within a factor of 2 of one another. However, at tighter thresholds the SPQE circuit contains significantly more CNOT gates than ADAPT VQE. For example, at $r_{\mathrm{H}-\mathrm{H}}=2.0 \AA, 114$ of the 169 operators used in SPQE are three body or higher, while ADAPT SD and ADAPT GSD of course contain only up to two-body operators. Consequently, the $\operatorname{SPQE}\left(\Omega=10^{-2}\right)$ circuit contains more CNOT gates (226768) than ADAPT VQE GSD (14776). As discussed in Appendix D, this large difference in CNOT count is due to the growth in cost to implement the exponential of $n$-body second-quantized operators as a function of $n$ (and the lack of circuit optimization). Nevertheless, it is important to note that the systems studied here are not large enough to draw definitive conclusions about the relative performance of SPQE and ADAPT VQE. For example, the pool of GSD for $\mathrm{H}_{6}$ contains 870 operators, a number significantly larger than the size of the full Hilbert space (200). This scenario is unlike most systems of interest, where the number of generalized singles and doubles is much less than the number of particle-hole operators. Unfortunately, our attempts to compare numbers for systems larger than $\mathrm{BeH}_{2}$ and $\mathrm{H}_{6}$ were unsuccessful due to the high computational cost of simulating ADAPT VQE.

A second aspect we investigate is the ability of the selected PQE approach to compactly represent wave functions for systems displaying strong correlation effects, where many-body methods commonly fail due to the breakdown of the mean-field approximation. We compare the performance of SPQE with the adaptive configuration interaction [90] (ACI) and DMRG using data generated in our recent benchmark study of hydrogen systems [81]. In this work, we characterize the resource requirements of a computational method $X$ with the accuracy volume $\left(\mathcal{V}_{X}\right)$, defined as the smallest number of parameters necessary to achieve a given energy error per electron (here taken to be $10^{-4} E_{h}$ (electron). We consider three $\mathrm{H}_{10}$ model systems: a one-dimensional (1D) linear chain, a 2D triangular lattice, and a 3D close-packed pyramid. Using a minimal STO6G basis and a 1 qubit to 1 spin-orbital mapping, the $1 \mathrm{D}$, $2 \mathrm{D}$, and $3 \mathrm{D} \mathrm{H}_{10}$ models are represented with $2^{20}$ computational basis states, but are restricted to 31752 and 15912 , and 15912 determinants, respectively, after accounting for spin and Abelian point-group symmetries.

Figure 6(a) displays the SPQE, ACI, and DMRG energy error as a function of classical variational parameters for the $1 \mathrm{D} \mathrm{H}_{10}$ system at a stretched bond length of $r_{\mathrm{H}-\mathrm{H}}=1.50 \AA$. DMRG, which is ideally suited to simulate gapped 1D systems, affords the most compact wave function for the $1 \mathrm{D}$ chain, with an accuracy volume of only 176 variational parameters $\left(\mathcal{V}_{\mathrm{DMRG}}^{1 \mathrm{D}}=\right.$ 176). The SPQE exponential ansatz is less compact than the DMRG matrix product state, with an accuracy volume $\mathcal{V}_{\mathrm{SPQE}}^{1 \mathrm{D}}=3510$ parameters. We observe that the ACI wave function - a linear ansatz built by selecting the determinants with the largest energy contributions - gives the least compact representation, such that $\mathcal{V}_{\mathrm{ACI}}^{1 \mathrm{D}}=22989$ at the same level of accuracy. When the ACI energy is augmented with a second-order perturbative correction - which accounts for determinants excluded from the wave-function expansion - a more compact ansatz is sufficient to achieve an energy error of $10^{-3} E_{h}$. This suggests that it might be valuable 

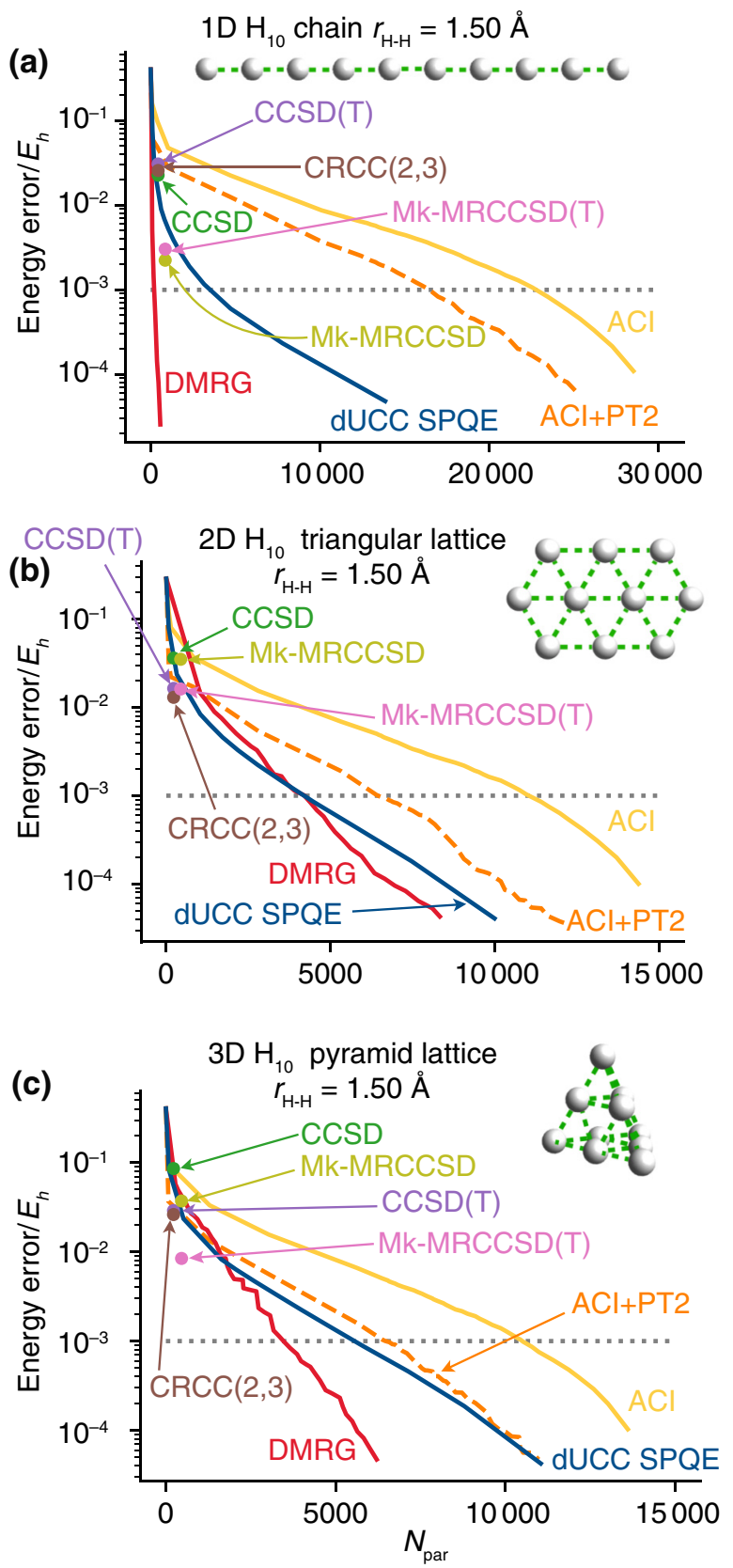

FIG. 6. Results for the $1 \mathrm{D}, 2 \mathrm{D}$, and $3 \mathrm{D} \mathrm{H}_{10}$ models use a STO$6 \mathrm{G}$ basis at $r_{\mathrm{H}-\mathrm{H}}=1.50 \AA$. Singlet ground-state energy errors relative to FCI as a function of the number of variational parameters $N_{\text {par }}$ for dUCC SPQE, ACI, and DMRG. Unsigned energy errors for methods with a fixed number of parameters (taken to be the number of cluster amplitudes) are shown by colored dots. Energy errors ACI with a second-order perturbative correction (ACI+PT2) are also shown by orange dotted lines. For ACI+PT2, $\operatorname{CCSD}(\mathrm{T}), \operatorname{CRCC}(2,3)$, and $\mathrm{Mk}-\operatorname{MRCCSD}(\mathrm{T})$, we only count the number of nonperturbative amplitudes. The accuracy volume threshold $\left(0.1 \mathrm{~m} E_{h}\right.$ per electron) is plotted as a gray dotted line.

to formulate a classical perturbative correction to the SPQE energy to help capture even more correlation energy.
Results for the 2D $\mathrm{H}_{10}$ lattice [Fig. 6(b)] show that SPQE yields a more compact representation than DMRG or ACI until an energy error of approximately $1 \mathrm{~m} E_{h}$. For the 2D system, the accuracy volume follows the order SPQE (4193) $\approx$ DMRG (4218) < ACI (11 122). At energy errors less than $1 \mathrm{~m} E_{h}$ DMRG still affords the most compact representation for the 2D system. Finally, for the 3D $\mathrm{H}_{10}$ pyramid lattice shown in Fig. 6(c), we find that DMRG yields the most compact representation $\left(\mathcal{V}_{\mathrm{DMRG}}^{3 \mathrm{D}}=3549\right)$, but not nearly by the same margin as for the 1D system. Again, the SPQE has a significantly more compact wave function than ACI such that $\mathcal{V}_{\mathrm{SPQE}}^{3 \mathrm{D}}=5544$ and $\mathcal{V}_{\mathrm{ACI}}^{3 \mathrm{D}}=$ 10519 . While further investigation of larger strongly correlated systems will be necessary, it is encouraging to see that SPQE performs similarly to or better than two stateof-the-art classical methodologies when applied to the 2D and $3 \mathrm{D} \mathrm{H}_{10}$ systems.

We have also included in Fig. 6 energy errors for several classical coupled-cluster variants. Specifically, we have included results from Ref. [81] for CC with single and double excitations [91] (CCSD), CCSD with perturbative triples [92] $[\mathrm{CCSD}(\mathrm{T})]$, and the completely renormalized $\mathrm{CC}$ with perturbative triples [12] [CRCC $(2,3)]$. These CC methods are (generally) more computationally affordable than the other theories. In the 2D and 3D systems, CCSD performs comparably to dUCC SPQE (with the same number of parameters), while $\operatorname{CCSD}(\mathrm{T})$ and $\operatorname{CRCC}(2,3)$ produce more accurate energies. However, all coupled-cluster energies are more than $10 \mathrm{~m} E_{h}$ off from the FCI energy. Figure 6 also reports results computed using Mukherjee MRCC with singles and doubles [93,94] (Mk-MRCCSD) and Mk-MRCCSD augmented with perturbative triples [95] [Mk-MRCCSD(T)] using an active space containing the highest occupied and lowest unoccupied Hartree-Fock orbitals. The Mk-MRCC results improve upon singlereference coupled-cluster methods at the cost of doubling the number of cluster amplitudes. The Mk-MRCC methods are particularly accurate for the 1D system, where they produce errors of the order of 2-3 $\mathrm{m} E_{h}$. Despite the improvement shown by the multireference CC methods, it is important to note that these methods have a computational cost that scales exponentially with the number of active space determinants.

\section{CONCLUSIONS}

In this work, we present a new NISQ-friendly algorithm - the PQE - to compute the ground state of a many-body problem using disentangled (factorized) unitary coupled-cluster trial states. The PQE approach consists of a nonlinear optimization problem whose solution requires the evaluation of projections of the Schrödinger equation onto a many-body basis (residual vector) but still gives energies that are a variational upper bound to the ground-state energy. We show how to efficiently evaluate 
the residual vector via measurement of simple expectation values, with a cost that is twice that of an energy evaluation (per element). For small molecular systems, we find that PQE and VQE with a fixed dUCCSD trial state converge to nearly identical energies; however, the number of residual evaluations required by $\mathrm{PQE}$ is smaller than that of the gradient evaluations needed by VQE. PQE shows similar resiliency as VQE and still converges more rapidly in the presence of stochastic noise.

To treat strongly correlated electrons, we introduce a selected variant of PQE in which the trial state is constructed iteratively by adding batches of important operators. The resulting SPQE algorithm can construct efficient unitary circuits like ADAPT VQE, but it requires orders of magnitude fewer residual element evaluations than gradient element evaluations for the latter. In SPQE, the selection of new operators is done according to the magnitude of the elements of the residual vector, and is performed by sampling a quantum state that directly encodes in its probability amplitudes the importance of the entire operator pool. Because the selection cost in SPQE is not affected by the size of the operator pool, the unitary can include operators of rank up to the total number of particles.

Finally, we compare the energy convergence with the number of parameters for $1 \mathrm{D}, 2 \mathrm{D}$, and $3 \mathrm{D} \mathrm{H}_{10}$ lattices using SPQE and two classical methods well suited to treat strong electronic correlation: the adaptive configuration interaction and the density matrix renormalization group. Given a target accuracy of up to approximately $1 \mathrm{~m} E_{h}$, we find that SPQE produces significantly more compact trial states for the 2D system than ACI and is comparable to DMRG. However, DMRG affords the most compact wavefunction parameterization in $1 \mathrm{D}$, for accuracies below 1 $\mathrm{m} E_{h}$ in $2 \mathrm{D}$, and by a much smaller margin, in 3D. Taken together, PQE and SPQE are very promising tools for studying many-body systems both in the strong and weak correlation regimes using NISQ hardware.

In summary, the PQE approach is a viable and more economical alternative to variational quantum algorithms. In its current formulation, PQE can be applied to any trial state generated by exponentiating a set of linearly independent operators with identity metric matrix, as it is the case for disentangled unitary coupled-cluster ansätze. For these trial states, methods to reduce the number of measurements and exploit symmetries [59,60,96] developed for VQE can likewise be used to improve PQE. Interesting extensions of $\mathrm{PQE}$ include a generalization to unitaries that may contain repeated operators, that use generalized excitation and de-excitation pools, and hardware-efficient ansätze. In particular, a promising research direction is the formulation of a selected PQE using a basis of general one- and two-body operators, which could yield trial states with lower circuit depth than the current formulation. Within the greater ecosystem of quantum algorithms, PQE could be used to determine initial guess amplitudes for subsequent optimization via VQE. Additionally, similarly to VQE, PQE can be used as an alternative to adiabatic approaches to prepare initial states for quantum phase estimation. Although we only explore applications of PQE for quantum many-body simulation, the framework outlined by Eqs. (3) and (4) can be used to solve a variety of eigenvalue problems. With appropriate modifications, for example, PQE could be employed to diagonalize covariance matrices (after quantum encoding [97,98]) for use in machine learning or data analysis. Moreover, because there is no requirement that the PQE (or SPQE) trial states have low entanglement, PQE could be used as an alternative to methods such as quantum principle analysis [98] or variational quantum state diagonalization [99]. Most importantly, the most immediate impact of PQE could be speeding up quantum computations on current or near-term devices.

\section{ACKNOWLEDGMENTS}

This work is supported by the U.S. Department of Energy under Award No. DE-SC0019374 and the NSF under Grant No. CHE-2038019. N.H.S. received support from a Molecular Sciences Software Institute Fellowship under NSF Grant No. ACI-1547580.

\section{APPENDIX A: GRADIENT OF THE PQE ENERGY}

In this section, we derive an expression for the PQE energy gradient. This result permits us to establish the equivalence of $\mathrm{PQE}$ and $\mathrm{VQE}$ for an exact $\mathrm{UCC}$ or dUCC ansatz, and to characterize the gradient when PQE is used to optimize approximate trial states. Consider the PQE energy expression using a UCC [Eq. (7)] or dUCC [Eq. (9)] ansatz $E_{\mathrm{PQE}}(\mathbf{t})=\left\langle\Phi_{0}\left|\hat{U}^{\dagger}(\mathbf{t}) \hat{H} \hat{U}(\mathbf{t})\right| \Phi_{0}\right\rangle$. The derivative of $E_{\mathrm{PQE}}(\mathbf{t})$ with respect to the parameter $t_{\mu}$ is given by

$$
\begin{aligned}
\frac{\partial E_{\mathrm{PQE}}(\mathbf{t})}{\partial t_{\mu}} & =2 \operatorname{Re}\left\langle\Phi_{0}\left|\hat{U}^{\dagger}(\mathbf{t}) \hat{H} \frac{\partial \hat{U}(\mathbf{t})}{\partial t_{\mu}}\right| \Phi_{0}\right\rangle \\
& =2 \operatorname{Re}\left\langle\Phi_{0}\left|\bar{H} \hat{U}^{\dagger}(\mathbf{t}) \frac{\partial \hat{U}(\mathbf{t})}{\partial t_{\mu}}\right| \Phi_{0}\right\rangle,
\end{aligned}
$$

where we have inserted the identity $\hat{U}^{\dagger}(\mathbf{t}) \hat{U}(\mathbf{t})=1$ to rewrite this expression in terms of $\bar{H}$.

Next, we consider the resolution of the identity

$$
1=\left|\Phi_{0}\right\rangle\left\langle\Phi_{0}\left|+\sum_{\Phi_{\mu} \in R}\right| \Phi_{\mu}\right\rangle\left\langle\Phi_{\mu}\left|+\sum_{\Phi_{\nu} \in S}\right| \Phi_{\nu}\right\rangle\left\langle\Phi_{\nu}\right|,
$$

where $R$ is the set of determinants for which the residual condition $r_{\mu}=0$ is enforced, while $S$ contains those determinants for which $r_{v}$ may not be equal to zero. After inserting this resolution of the identity into Eq. (A1) between 
$\bar{H}$ and $\hat{U}^{\dagger}(\mathbf{t})$ and simplifying the resulting expression, we may express the gradient as

$$
\begin{aligned}
\frac{\partial E_{\mathrm{PQE}}(\mathbf{t})}{\partial t_{\mu}} & =2 E_{\mathrm{PQE}} \operatorname{Re}\left\langle\Phi_{0}\left|\hat{U}^{\dagger}(\mathbf{t}) \frac{\partial \hat{U}(\mathbf{t})}{\partial t_{\mu}}\right| \Phi_{0}\right\rangle \\
& +2 \operatorname{Re} \sum_{\Phi_{\mu} \in R} r_{\mu}^{*}\left\langle\Phi_{\mu}\left|\hat{U}^{\dagger}(\mathbf{t}) \frac{\partial \hat{U}(\mathbf{t})}{\partial t_{\mu}}\right| \Phi_{0}\right\rangle \\
& +2 \operatorname{Re} \sum_{\Phi_{\nu} \in S} r_{\nu}^{*}\left\langle\Phi_{\nu}\left|\hat{U}^{\dagger}(\mathbf{t}) \frac{\partial \hat{U}(\mathbf{t})}{\partial t_{\mu}}\right| \Phi_{0}\right\rangle .
\end{aligned}
$$

The first term in this expression is null since from $\left\langle\Phi_{0}\left|\hat{U}^{\dagger}(\mathbf{t}) \hat{U}(\mathbf{t})\right| \Phi_{0}\right\rangle=1$ we can show that the coefficient that multiplies the energy is null:

$$
\frac{\partial\left\langle\Phi_{0}\left|\hat{U}^{\dagger}(\mathbf{t}) \hat{U}(\mathbf{t})\right| \Phi_{0}\right\rangle}{\partial t_{\mu}}=2 \operatorname{Re}\left\langle\Phi_{0}\left|\hat{U}^{\dagger}(\mathbf{t}) \frac{\partial \hat{U}(\mathbf{t})}{\partial t_{\mu}}\right| \Phi_{0}\right\rangle=0 .
$$

The second term in Eq. (A3) is null due to the residual condition. Applying these two simplifications we arrive at the gradient expression [Eq. (10)] reported in Sec. II B, containing only the last term of Eq. (A3). The term $\left\langle\Phi_{\nu}\left|\hat{U}^{\dagger}(\mathbf{t}) \partial \hat{U}(\mathbf{t}) / \partial t_{\mu}\right| \Phi_{0}\right\rangle$ that multiplies the residual is generally non-null for both the UCC and dUCC ansätze. In the case of $\mathrm{dUCC}$, the term corresponding to the derivative with respect to the $i$ th amplitude $t_{\mu_{i}}$ is given by

$$
\begin{aligned}
& \left\langle\Phi_{v}\left|\hat{U}^{\dagger}(\mathbf{t}) \frac{\partial \hat{U}(\mathbf{t})}{\partial t_{\mu_{i}}}\right| \Phi_{0}\right\rangle \\
& =\left\langle\Phi_{\nu}\right| e^{-t_{\mu_{\mathrm{Nop}}} \hat{\kappa}_{\mu_{\mathrm{Op}}}} \cdots e^{-t_{\mu_{i+1}} \hat{\kappa}_{\mu_{i+1}}} \hat{\kappa}_{\mu_{i}} e^{t_{\mu_{i+1}} \hat{\kappa}_{\mu_{i+1}}} \ldots \\
& \times e^{t_{\mu_{N_{\mathrm{op}}}} \hat{\kappa}_{\mu_{N_{\mathrm{op}}}}}\left|\Phi_{0}\right\rangle \text {. }
\end{aligned}
$$

The states to the left and right of the operator $\hat{\kappa}_{\mu_{i}}$ are general many-body states that potentially span the entire Hilbert space, and, therefore, this quantity is generally nonnull. For completeness, we also report the same expression for the case of traditional UCC, which may be obtained using the derivative of the exponential map:

$$
\left\langle\Phi_{\nu}\left|\hat{U}^{\dagger}(\mathbf{t}) \frac{\partial \hat{U}(\mathbf{t})}{\partial t_{\mu_{i}}}\right| \Phi_{0}\right\rangle=\int_{0}^{1} d x\left\langle\Phi_{\nu}\left|e^{(x-1) \hat{\sigma}} \hat{\kappa}_{\mu_{i}} e^{(1-x) \hat{\sigma}}\right| \Phi_{0}\right\rangle .
$$

\section{APPENDIX B: DERIVATION OF THE dUCC PQE UPDATE EQUATIONS}

In this section, we provide a derivation of the amplitude update equation [Eq. (11)] based on a quasi-Newton method for solving general nonlinear equations. To find update equations that relate new amplitudes $\left(\mathbf{t}^{(n+1)}\right)$ to the previous set $\left(\mathbf{t}^{(n)}\right)$, we expand the residual equation [Eq. (4)] as a Taylor series centered around the current amplitude vector displaced by an amount $\Delta \mathbf{t}^{(n+1)}=$ $\mathbf{t}^{(n+1)}-\mathbf{t}^{(n)}$,

$$
\begin{aligned}
r_{\mu}\left(\mathbf{t}^{(n+1)}\right) & =r_{\mu}\left(\mathbf{t}^{(n)}+\Delta \mathbf{t}^{(n+1)}\right) \\
& =r_{\mu}\left(\mathbf{t}^{(n)}\right)+\sum_{\nu} J_{\mu \nu}\left(\mathbf{t}^{(n)}\right) \Delta t_{v}^{(n+1)}+\cdots
\end{aligned}
$$

where $J$ is the Jacobian matrix, defined as $J_{\mu \nu}(\mathbf{t})=$ $\partial r_{\mu}(\mathbf{t}) / \partial t_{\nu}$. To avoid computing and inverting the Jacobian, we seek a diagonal approximation to $J$. We first note that, for both the conventional UCC and the dUCC ansätze, the similarity transformed Hamiltonian expanded up to linear terms is of the form

$$
\hat{U}^{\dagger}(\mathbf{t}) \hat{H} \hat{U}(\mathbf{t})=\hat{H}+\sum_{i} t_{\mu_{i}}\left[\hat{H}, \hat{\kappa}_{\mu_{i}}\right]+\mathcal{O}\left(|\mathbf{t}|^{2}\right) .
$$

We now invoke the Møller-Plesset partitioning of the Hamiltonian and assume that the spin orbitals are canonical (i.e., they diagonalize the Hartree-Fock operator), allowing us to write $\hat{H}=\hat{F}^{(0)}+\hat{V}^{(1)}$, where $\hat{F}^{(0)}=$ $\sum_{p} \epsilon_{p} \hat{a}_{p}^{\dagger} \hat{a}_{p}$ is a zeroth-order diagonal one-body operator, and $\hat{V}^{(1)}$ is a first-order operator that contains two-body terms. Approximating the Jacobian with the first two terms of Eq. (B2) and retaining only the zeroth-order contributions, we obtain the diagonal approximation

$$
J_{\mu \nu}(\mathbf{t})=\left\langle\Phi_{\mu}\left|\left[\hat{F}^{(0)}, \hat{\kappa}_{\nu}\right]\right| \Phi_{0}\right\rangle=-\Delta_{\mu} \delta_{\mu \nu}
$$

where $\Delta_{\mu}=\epsilon_{i}+\epsilon_{j}+\cdots-\epsilon_{a}-\epsilon_{b} \ldots$ is a standard Møller-Plesset denominator corresponding to the excitation operator $\hat{\kappa}_{\mu}=\hat{a}_{a}^{\dagger} \hat{a}_{b}^{\dagger} \cdots \hat{a}_{j} \hat{a}_{i}-$ H.c. Inserting the diagonal Jacobian in the expanded residual we obtain

$$
r_{\mu}\left(\mathbf{t}^{(n+1)}\right)=r_{\mu}\left(\mathbf{t}^{(n)}\right)-\Delta_{\mu} \Delta t_{\mu}^{(n+1)}
$$

which when solved for $r_{\mu}\left(\mathbf{t}^{(n+1)}\right)=0$ yields the update equation [Eq. (11)].

\section{APPENDIX C: ADDITIONAL NUMERICAL COMPARISON OF PQE AND VQE}

Here we provide additional numerical comparison for $\mathrm{PQE}$ and VQE. Table II shows numerics for ground-state energy convergence of $\mathrm{BeH}_{2}$ using dUCCSD PQE and dUCCSD VQE. Figure 7 shows the energy convergence of various hydrogen chain systems with respect to the norm of the residual vector (for PQE) and gradient vector (for VQE). For both Table II and Fig. 7, trends similar to those seen in Fig. 3 are observed. 
TABLE II. Comparison of the convergence of the ground-state energy of linear $\mathrm{BeH}_{2}$ computed with PQE and VQE using a disentangled UCCSD trial state. For a method $X=\mathrm{PQE}$ or VQE, this table reports the total energy $\left(E_{X}\right.$, in $\left.E_{h}\right)$, the energy change from the previous to the current iteration $\left(E_{X}^{(n)}-E_{X}^{(n-1)}\right)$, the number of residual or gradient evaluations $\left(N_{X}\right)$, and the norm of the residual or gradient $(\|\cdot\|)$. The FCI energies at 1.0 and $2.0 \AA$ are -15.65068726 and $-15.60861964 E_{h}$, respectively. All calculations use a STO-6G basis.

\begin{tabular}{|c|c|c|c|c|c|c|c|c|}
\hline Iteration & $E_{\mathrm{PQE}}$ & $E_{\mathrm{PQE}}^{(n)}-E_{\mathrm{PQE}}^{(n-1)}$ & $N_{\mathrm{PQE}}^{r-\text { eval }}$ & $\left\|\mathbf{r}_{\mathrm{PQE}}\right\|$ & $E_{\mathrm{VQE}}$ & $E_{\mathrm{VQE}}^{(n)}-E_{\mathrm{VQE}}^{(n-1)}$ & $N_{\mathrm{VQE}}^{g-\text { eval }}$ & $\left\|\mathbf{g}_{\mathrm{VQE}}\right\|$ \\
\hline \multicolumn{9}{|c|}{$\mathrm{BeH}_{2}\left(r_{\mathrm{Be}-\mathrm{H}}=1.0 \AA\right) \mathrm{UCCSD}$} \\
\hline 1 & -15.6480381687 & -0.0233010440 & 1 & 0.2184453066 & -15.6448482964 & -0.0201111716 & 3 & 0.3114581416 \\
\hline 2 & -15.6500971948 & -0.0020590260 & 2 & 0.0613327467 & -15.6478816410 & -0.0030333446 & 5 & 0.1481817759 \\
\hline 3 & -15.6504069826 & -0.0003097878 & 3 & 0.0088860708 & -15.6494462890 & -0.0015646480 & 7 & 0.1065575796 \\
\hline 4 & -15.6504328168 & -0.0000258343 & 4 & 0.0026511924 & -15.6499421388 & -0.0004958498 & 9 & 0.0634937794 \\
\hline 5 & -15.6504349587 & -0.0000021418 & 5 & 0.0005328227 & -15.6500470587 & -0.0001049199 & 10 & 0.0893255742 \\
\hline 6 & -15.6504350041 & -0.0000000454 & 6 & 0.0000763318 & -15.6501955946 & -0.0001485359 & 11 & 0.0657024179 \\
\hline 7 & -15.6504350044 & -0.0000000003 & 7 & 0.0000086198 & -15.6502874046 & -0.0000918100 & 12 & 0.0690985413 \\
\hline 8 & & & & & -15.6503399456 & -0.0000525410 & 13 & 0.0453884569 \\
\hline 9 & & & & & -15.6503962522 & -0.0000563066 & 14 & 0.0249260915 \\
\hline 10 & & & & & -15.6504228495 & -0.0000265973 & 16 & 0.0137252110 \\
\hline 11 & & & & & -15.6504302548 & -0.0000074053 & 18 & 0.0073189453 \\
\hline 12 & & & & & -15.6504341054 & -0.0000038506 & 20 & 0.0037260965 \\
\hline 13 & & & & & -15.6504346315 & -0.0000005261 & 22 & 0.0023579786 \\
\hline 14 & & & & & -15.6504348973 & -0.0000002657 & 24 & 0.0014145610 \\
\hline 15 & & & & & -15.6504349615 & -0.0000000642 & 26 & 0.0008586922 \\
\hline 16 & & & & & -15.6504349766 & -0.0000000151 & 28 & 0.0006710048 \\
\hline 17 & & & & & -15.6504349959 & -0.0000000193 & 29 & 0.0003518429 \\
\hline 18 & & & & & -15.6504350027 & -0.0000000068 & 31 & 0.0001962939 \\
\hline 19 & & & & & -15.6504350038 & -0.0000000011 & 33 & 0.0001228873 \\
\hline 20 & & & & & -15.6504350044 & -0.0000000005 & 34 & 0.0000505365 \\
\hline 21 & & & & & -15.6504350045 & -0.0000000001 & 36 & 0.0000433400 \\
\hline 22 & & & & & -15.6504350047 & -0.0000000002 & 37 & 0.0000200500 \\
\hline \multicolumn{9}{|c|}{$\mathrm{BeH}_{2}\left(r_{\mathrm{Be}-\mathrm{H}}=2.0 \AA\right) \mathrm{UCCSD}$} \\
\hline 1 & -15.5888292373 & -0.0721251319 & 1 & 0.2551300809 & -15.5797540529 & -0.0630499476 & 3 & 0.7559489104 \\
\hline 2 & -15.6022316771 & -0.0134024398 & 2 & 0.1072484292 & -15.5993381767 & -0.0195841238 & 5 & 0.2319720501 \\
\hline 3 & -15.6055237786 & -0.0032921016 & 3 & 0.0175246100 & -15.6027545167 & -0.0034163400 & 7 & 0.1024944422 \\
\hline 4 & -15.6056880097 & -0.0001642310 & 4 & 0.0076659595 & -15.6041840035 & -0.0014294868 & 9 & 0.1471217958 \\
\hline 5 & -15.6057527283 & -0.0000647187 & 5 & 0.0051078803 & -15.6050561955 & -0.0008721920 & 10 & 0.0754837786 \\
\hline 6 & -15.6058044053 & -0.0000516769 & 6 & 0.0012840244 & -15.6052847256 & -0.0002285301 & 12 & 0.0580899178 \\
\hline 7 & -15.6058068352 & -0.0000024300 & 7 & 0.0002042506 & -15.6055945458 & -0.0003098202 & 13 & 0.0291205752 \\
\hline 8 & -15.6058068368 & -0.0000000016 & 8 & 0.0000663613 & -15.6056501930 & -0.0000556471 & 15 & 0.0302968175 \\
\hline 9 & -15.6058068335 & +0.0000000033 & 9 & 0.0000122091 & -15.6057330619 & -0.0000828689 & 16 & 0.0197063516 \\
\hline 10 & -15.6058068336 & -0.0000000000 & 10 & 0.0000040077 & -15.6057681510 & -0.0000350891 & 18 & 0.0186569595 \\
\hline 11 & & & & & -15.6058033733 & -0.0000352223 & 19 & 0.0069816557 \\
\hline 12 & & & & & -15.6058052286 & -0.0000018553 & 21 & 0.0045627712 \\
\hline 13 & & & & & -15.6058057676 & -0.0000005391 & 23 & 0.0036888638 \\
\hline 14 & & & & & -15.6058065558 & -0.0000007882 & 24 & 0.0021623560 \\
\hline 15 & & & & & -15.6058067218 & -0.0000001661 & 26 & 0.0015523193 \\
\hline 16 & & & & & -15.6058068503 & -0.0000001285 & 27 & 0.0003628532 \\
\hline 17 & & & & & -15.6058068547 & -0.0000000044 & 29 & 0.0002829316 \\
\hline 18 & & & & & -15.6058068601 & -0.0000000054 & 30 & 0.0000872433 \\
\hline 19 & & & & & -15.6058068604 & -0.0000000003 & 32 & 0.0000660842 \\
\hline 20 & & & & & -15.6058068607 & -0.0000000002 & 33 & 0.0000166869 \\
\hline
\end{tabular}

\section{APPENDIX D: FORMAL COMPARISON OF PQE AND VQE}

We begin by assuming the case of a fixed ansatz, and since in this case state preparation costs are the same, our analysis focuses on how many measurements are necessary to compute the gradients and residuals in the iterative optimization procedure.

The preferred approach to minimize the energy in VQE is via gradient-based algorithms. The gradient of the dUCC energy with respect to a cluster amplitude $t_{\mu}$ is given by 

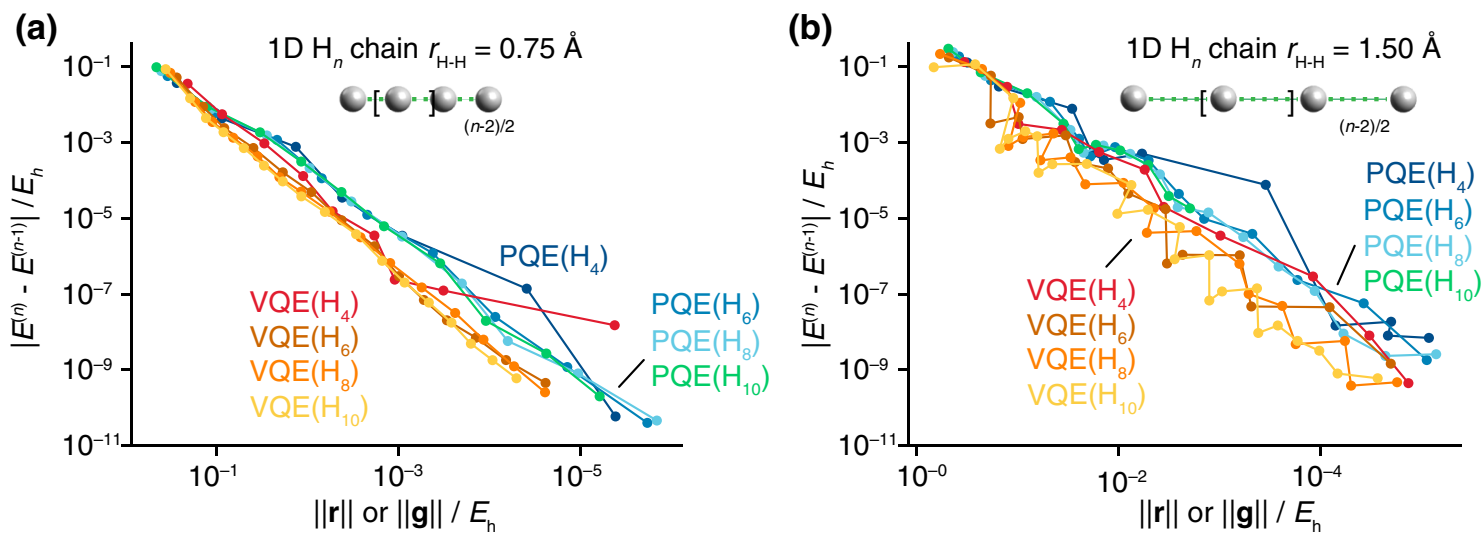

FIG. 7. dUCCSD energy convergence for linear $\mathrm{H}_{4}-\mathrm{H}_{10}$ chains in a STO-6G basis at (a) $r_{\mathrm{H}-\mathrm{H}}=0.75 \AA$ and (b) $r_{\mathrm{H}-\mathrm{H}}=1.50 \AA$. Here $\left|E^{(n)}-E^{(n-1)}\right|$ is the absolute value of the energy change between subsequent iterations. Both plots compare PQE versus VQE convergence with respect to the norm of the residual vector (for PQE) or the norm of the gradient vector (for VQE).

$$
\frac{\partial E_{\mathrm{VQE}}(\mathbf{t})}{\partial t_{\mu}}=2 \operatorname{Re}\left\langle\Phi_{0}\left|\hat{U}^{\dagger}(\mathbf{t}) \hat{H} \frac{\partial \hat{U}(\mathbf{t})}{\partial t_{\mu}}\right| \Phi_{0}\right\rangle
$$

Equation (D1) has the form of an off-diagonal matrix element of the Hamiltonian, and Romero et al. [77] showed that it may be measured on a quantum device using one ancilla qubit and 4 times the cost of measuring the energy. With this algorithm, computing the gradient vector so that the 2-norm of the error is within precision $\bar{\epsilon}_{\text {grad }}$ requires a total number of measurements $\left(m_{\text {grad }}\right)$ that is bound by the inequality [77]

$$
m_{\text {grad }} \leq 4 N_{\text {par }} \frac{\left(\sum_{\ell}\left|h_{\ell}\right|\right)^{2}}{\bar{\epsilon}_{\text {grad }}^{2}} .
$$

The total number of measurements, and proportionally the runtime, required for a $\mathrm{VQE}$ calculation is dominated by the computation of the gradient vector and it is proportional to the number of gradient vector evaluations $\left(N_{\text {grad }}^{\mathrm{VQE}}\right)$ required to converge the energy. This implies that the total number of $\mathrm{VQE}$ measurements $\left(m_{\mathrm{VQE}}\right)$ is bounded approximately by

$$
m_{\mathrm{VQE}} \leq N_{\mathrm{grad}}^{\mathrm{VQE}} m_{\mathrm{grad}} .
$$

This estimate ignores the evaluation of the VQE energy, which has a cost inferior to that of computing one element of the gradient vector. More recently, Kottmann et al. [64] showed that the analytic gradient may be computed with a cost essentially equal to that of two energy evaluations using the so-called parameter-shift rule [63]. This procedure avoids the use of an ancilla qubit and the number of measurements required still satisfies the bound expressed in Eq. (D2).

In the case of PQE, the number of measurements $m_{\text {res }}$ needed to compute the residual vector with precision $\bar{\epsilon}_{\text {res }}$ has an upper bound given by

$$
m_{\text {res }} \leq 3 N_{\text {par }} \frac{\left(\sum_{\ell}\left|h_{\ell}\right|\right)^{2}}{\bar{\epsilon}_{\text {res }}^{2}} .
$$

This estimate takes into account the fact that the residual can be evaluated as the sum of three terms (with different prefactors), and that $E_{0}$ in Eq. (14) only needs to be measured once. The total number of PQE measurements $\left(m_{\mathrm{PQE}}\right)$ is bounded by

$$
m_{\mathrm{PQE}} \leq N_{\mathrm{res}}^{\mathrm{PQE}} m_{\text {res }},
$$

where $N_{\text {res }}^{\mathrm{PQE}}$ is the number of PQE residual vector evaluations. Assuming that the energy gradients in $\mathrm{VQE}$ and the residual in PQE are measured with the same precision $\left(\bar{\epsilon}_{\text {grad }} \approx \bar{\epsilon}_{\text {res }}\right)$, we estimate that $m_{\text {res }} \approx \frac{3}{4} m_{\text {grad. }}$. This result suggests that $\mathrm{PQE}$ should have a similar or perhaps slightly smaller cost per iteration than VQE. However, a more important factor in determining the relative performance of VQE and PQE is the number of residual or gradient evaluations required, which as shown in Sec. III, favors PQE over VQE.

A detailed comparison of the adaptive variants of VQE (e.g., ADAPT VQE) and PQE is more complex due to the significant differences in the form of the ansatz and the selection procedure used in these two methods. At iteration $k$, the ADAPT VQE approach selects the operator $\hat{\kappa}_{\mu}$ with the largest absolute energy gradient. This selection scheme requires evaluating the gradient $g_{\mu}^{(k)}$ for all the operators in the pool,

$$
g_{\mu}^{(k)}=\left\langle\Psi^{(k)}\left|\left[\hat{H}, \hat{\kappa}_{\mu}\right]\right| \Psi^{(k)}\right\rangle .
$$

Because most subterms of the Hamiltonian will commute with $\hat{\kappa}_{\mu}$, a relatively small number of $\left\langle\hat{\kappa}_{\mu} \hat{O}_{\ell}\right\rangle$ and $\left\langle\hat{O}_{\ell} \hat{\kappa}_{\mu}\right\rangle$ 
terms need to be measured. Both $\hat{H}$ and the operator pool $\left\{\hat{\kappa}_{\mu}\right\}$ contain of the order $N^{4}$ elements (assuming a pool of general one- and two-body operators).

It has recently been pointed out [67] however that if the quantity $\left[\hat{H}, \hat{\kappa}_{\mu}\right]$ is decomposed in terms of the reduced density matrices then one can determine the pool gradients [Eq. (D6)] with the evaluation of a number of Pauli terms that scales as $N^{6}$ (again assuming a pool of general oneand two-body operators). In ADAPT VQE this cost must then be multiplied by the number of iterations performed. It is important to note that the ADAPT VQE macroiteration convergence threshold $\epsilon_{\alpha}=10^{-\alpha}$ is based on the norm of the vector of pool gradients [Eq. (D6)], such that ADAPT VQE is considered converged when $\left\|\mathbf{g}_{\text {pool }}\right\| \leq \epsilon_{\alpha}$. The number of measurements of the approximate residual vector $(|\tilde{r}\rangle)$ for the purpose of selection in SPQE is a parameter of a computation. In Appendix E, we show that a probabilistic estimate for the number of measurements required to converge SPQE with a threshold of $\Omega$ is of the order of $(\Delta t \Omega)^{-2}$.

A trade-off of using three- and higher-body operators in SPQE is a greater circuit depth since an operator $\hat{\kappa}_{i j \cdots}^{a b \cdots}$ of many-body rank $n$ becomes a sum of $2^{2 n-1}$ Pauli strings after Jordan-Wigner mapping to the qubit basis. Since all Pauli strings that are generated in this mapping commute, the unitary $\exp \left(\theta \hat{\kappa}_{i j \cdots}^{a b \cdots}\right)$ can be written as the product of $2^{2 n-1}$ exponentials of operators containing Pauli strings of length $2 n$.

To analyze the compromise between the rank of the operator pool and the compactness of the ansatz in ADAPT VQE and SPQE, we consider the example of a three-body operator $\exp \left(t \hat{\kappa}_{i j k}^{a b c}\right)$. In ADAPT VQE this term may be approximated using general two-body operators as

$$
e^{t \hat{\kappa}_{i j k}^{a b c}}=e^{t\left[\hat{\kappa}_{i j}^{a e}, \hat{\kappa}_{k e}^{b c}\right]} \approx e^{t \hat{\kappa}_{i j}^{a e}} e^{t \hat{\kappa}_{k e}^{b c}} e^{-t \hat{\kappa}_{i j}^{a e}} e^{-t \hat{\kappa}_{k e}^{b c}},
$$

where the last term is a lowest-order Trotter approximation of the exponential of the commutator $\left[\hat{\kappa}_{i j}^{a e}, \hat{\kappa}_{k e}^{b c}\right]$ (with $e \neq a, b, c)$. The last term in Eq. (D7) is implemented as a circuit that contains four different parameters and a product of 32 exponentials of Pauli strings of length four. The same three-body excitation is represented in SPQE using a single parameter and a longer circuit as a product of 32 exponentials of Pauli strings of length six. This comparison suggests, in accordance with the results of our study, that higher-body operators are represented less efficiently with an arbitrary particle-hole operator pool than a general singles and doubles operator pool.

\section{APPENDIX E: REDUCED-COST ESTIMATION OF THE APPROXIMATE RESIDUAL IN SELECTED PQE}

This appendix explores various methods to reduce the number of measurements $M$ required to compute the approximate residuals $\tilde{r}_{\mu}$ used in the selected PQE method (see Sec. II E). Selection requires the identification of the elements of the approximate residual that corresponds to projections onto excited determinants. However, for small values of $\Delta t$, the state $|\tilde{r}\rangle$ is dominated by the reference determinant $\Phi_{0}$, and, consequently, the measurement of important missing excitations may become inefficient. In practice, one can consider an alternative convergence criterion for SPQE based on performing a fixed number of measurements $M_{\Omega}$ on the state $|\tilde{r}\rangle$. In such an approach, at each iteration $k$, the operators $\hat{\kappa}_{\mu}$ whose corresponding determinants $\left|\Phi_{\mu}\right\rangle$ are measured at least once (over all $M_{\Omega}$ measurements) are added to $\mathcal{A}$. Because the residual magnitudes go to zero as the eigenstate is better approximated in successive $k$ iterations, it becomes increasingly unlikely that any determinants besides the reference $\left|\Phi_{0}\right\rangle$ will be measured. The SPQE algorithm can then be considered converged when all $M_{\Omega}$ measurements yield the reference state. Starting from Eqs. (16) and (17), and making the assumption that at convergence only a single determinant $\Phi_{\mu}$ is measured that is not the reference (i.e., $\sum_{\mu} N_{\mu}=1$ ), one can use a number of measurements

$$
M_{\Omega}=\frac{1}{\Delta t^{2} \Omega^{2}}
$$

to probabilistically test convergence of the residual vector to within the threshold $\Omega$. In practice, we find that using Eq. (E1) works well compared to the exact threshold given in Eq. (17). Figure 8 compares the energy convergence with the number of selected operators or parameters using both convergence criterion corresponding to the same value of $\Omega$.

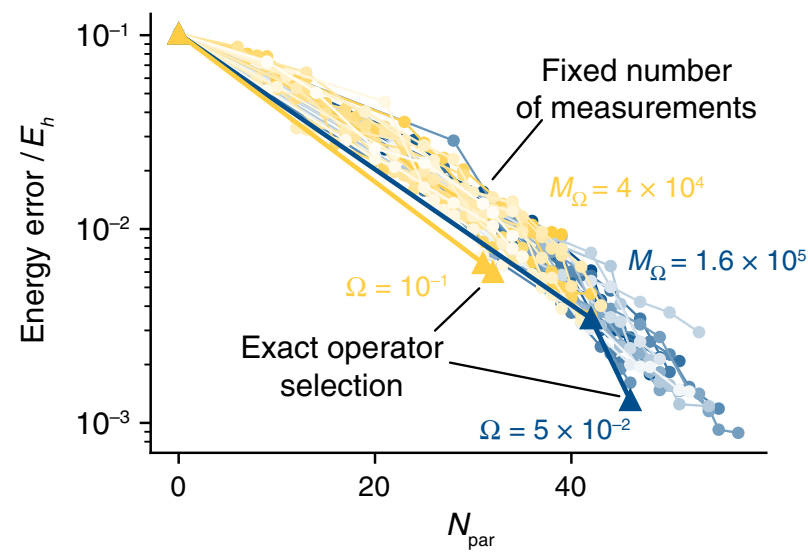

FIG. 8. SPQE energy convergence for $\mathrm{H}_{6}$ at a separation of $r_{\mathrm{H}-\mathrm{H}}=1.0 \AA$, with $\Delta t=0.05 \mathrm{au}$. Data sets with triangular markers denote exact operator selection and convergence via Eqs. (16) and (17) for $\Omega=1.0 \times 10^{-1}$ (yellow) and $\Omega=5.0 \times 10^{-2}$ (dark blue). Data sets with dots denote operator selection and convergence with a fixed number of measurements $M_{\Omega}=4.0 \times 10^{4}$ (yellow) and $M_{\Omega}=1.6 \times 10^{5}$ (dark blue) calculated via Eq. (E1) for the corresponding $\Omega$ values. 
[1] S. Lloyd, Universal quantum simulators, Science 273, 1073 (1996).

[2] A. Aspuru-Guzik, A. D. Dutoi, P. J. Love, and M. HeadGordon, Simulated quantum computation of molecular energies, Science 309, 1704 (2005).

[3] A. Kandala, A. Mezzacapo, K. Temme, M. Takita, M. Brink, J. M. Chow, and J. M. Gambetta, Hardware-efficient variational quantum eigensolver for small molecules and quantum magnets, Nature 549, 242 (2017).

[4] A. Kandala, K. Temme, A. D. Córcoles, A. Mezzacapo, J. M. Chow, and J. M. Gambetta, Error mitigation extends the computational reach of a noisy quantum processor, Nature 567, 491 (2019).

[5] S. R. White, Density Matrix Formulation for Quantum Renormalization Groups, Phys. Rev. Lett. 69, 2863 (1992).

[6] B. Huron, J.-P. Malrieu, and P. Rancurel, Iterative perturbation calculations of ground and excited state energies from multiconfigurational zeroth order wavefunctions, J. Chem. Phys. 58, 5745 (1973).

[7] R. J. Buenker and S. D. Peyerimhoff, Individualized configuration selection in ci calculations with subsequent energy extrapolation, Theor. Chim. Acta 35, 33 (1974).

[8] G. H. Booth, A. J. W. Thom, and A. Alavi, Fermion monte carlo without fixed nodes: A game of life, death, and annihilation in slater determinant space, J. Chem. Phys. 131, 054106 (2009).

[9] F. Coester and H. Kümmel, Short-range correlations in nuclear wave functions, Nuc. Phys. 17, 477 (1960).

[10] J. Ćížek, On the correlation problem in atomic and molecular systems. calculation of wavefunction components in ursell-type expansion using quantum-field theoretical methods, J. Chem. Phys. 45, 4256 (1966).

[11] P. Piecuch, S. Zarrabian, J. Paldus, and J. Čižek, Coupledcluster approaches with an approximate account of triexcitations and the optimized-inner-projection technique. II. Coupled-cluster results for cyclic-polyene model systems, Phys. Rev. B 42, 3351 (1990).

[12] P. Piecuch and M. Włoch, Renormalized coupled-cluster methods exploiting left eigenstates of the similaritytransformed hamiltonian, J. Chem. Phys. 123, 224105 (2005).

[13] P. A. Limacher, P. W. Ayers, P. A. Johnson, S. De Baerdemacker, D. Van Neck, and P. Bultinck, A new mean-field method suitable for strongly correlated electrons: Computationally facile antisymmetric products of nonorthogonal geminals, J. Chem. Theory Comput. 9, 1394 (2013).

[14] I. W. Bulik, T. M. Henderson, and G. E. Scuseria, Can single-reference coupled cluster theory describe static correlation?, J. Chem. Theory Comput. 11, 3171 (2015).

[15] J. Ćížek, On the use of the cluster expansion and the technique of diagrams in calculations of correlation effects in atoms and molecules, Adv. Chem. Phys 14, 35 (1969).

[16] I. Lindgren, A coupled-cluster approach to the many-body perturbation theory for open-shell systems, Int. J. Quantum Chem. 14, 33 (1978).

[17] B. Jeziorski and H. J. Monkhorst, Coupled-cluster method for multideterminantal reference states, Phys. Rev. A 24, 1668 (1981).
[18] D. I. Lyakh, M. Musiał, V. F. Lotrich, and R. J. Bartlett, Multireference nature of chemistry: The coupled-cluster view, Chem. Rev. 112, 182 (2012).

[19] A. Köhn, M. Hanauer, L. A. Mück, T.-C. Jagau, and J. Gauss, State-specific multireference coupled-cluster theory, Wiley Interdiscip. Rev.: Comput. Mol. Sci. 3, 176 (2013).

[20] F. A. Evangelista, Perspective: Multireference coupled cluster theories of dynamical electron correlation, J. Chem. Phys. 149, 030901 (2018).

[21] R. P. Feynman, Simulating physics with computers, Int. J. Theor. Phys 21, 133 (1982).

[22] A. Y. Kitaev, Quantum measurements and the abelian stabilizer problem, arXiv:9511026 (1995).

[23] D. S. Abrams and S. Lloyd, Simulation of ManyBody Fermi Systems on a Universal Quantum Computer, Phys. Rev. Lett. 79, 2586 (1997).

[24] D. S. Abrams and S. Lloyd, Quantum Algorithm Providing Exponential Speed Increase for Finding Eigenvalues and Eigenvectors, Phys. Rev. Lett. 83, 5162 (1999).

[25] H. F. Trotter, On the product of semi-groups of operators, Proc. Am. Math. Soc. 10, 545 (1959).

[26] M. Suzuki, Improved trotter-like formula, Physics Letters A 180, 232 (1993).

[27] V. von Burg, G. H. Low, T. Häner, D. S. Steiger, M. Reiher, M. Roetteler, and M. Troyer, Quantum computing enhanced computational catalysis, arXiv:2007.14460 (2020).

[28] J. Lee, D. Berry, C. Gidney, W. J. Huggins, J. R. McClean, $\mathrm{N}$. Wiebe, and R. Babbush, Even more efficient quantum computations of chemistry through tensor hypercontraction, arXiv:2011.03494 (2020).

[29] R. Babbush, N. Wiebe, J. R. McClean, J. McClain, H. Neven, and G. K.-L. Chan, Low-Depth Quantum Simulation of Materials, Phys. Rev. X 8, 011044 (2018).

[30] M. Reiher, N. Wiebe, K. M. Svore, D. Wecker, and M. Troyer, Elucidating reaction mechanisms on quantum computers, Proc. Natl. Acad. Sci. U.S.A 114, 7555 (2017).

[31] J. Kempe, A. Kitaev, and O. Regev, The complexity of the local hamiltonian problem, SIAM J. Comput. 35, 1070 (2006).

[32] J. Preskill, Quantum computing in the nisq era and beyond, Quantum 2, 79 (2018).

[33] A. Peruzzo, J. R. McClean, P. Shadbolt, M.-H. Yung, X.-Q. Zhou, P. J. Love, A. Aspuru-Guzik, and J. L. O'Brien, A variational eigenvalue solver on a photonic quantum processor., Nat. Commun. 5, 4213 (2014).

[34] M.-H. Yung, J. Casanova, A. Mezzacapo, J. R. McClean, L. Lamata, A. Aspuru-Guzik, and E. Solano, From transistor to trapped-ion computers for quantum chemistry, Sci. Rep. 4, 3589 (2014).

[35] J. R. McClean, J. Romero, R. Babbush, and A. AspuruGuzik, The theory of variational hybrid quantum-classical algorithms, New J. Phys. 18, 023023 (2016).

[36] H. R. Grimsley, S. E. Economou, E. Barnes, and N. J. Mayhall, An adaptive variational algorithm for exact molecular simulations on a quantum computer, Nat. Commun. 10, 1 (2019).

[37] P. J. J. O’Malley, R. Babbush, I. D. Kivlichan, J. Romero, J. R. McClean, R. Barends, J. Kelly, P. Roushan, A. Tranter, and N. Ding et al., Scalable Quantum Simulation of Molecular Energies, Phys. Rev. X 6, 031007 (2016). 
[38] J. I. Colless, V. V. Ramasesh, D. Dahlen, M. S. Blok, M. Kimchi-Schwartz, J. R. McClean, J. Carter, W. De Jong, and I. Siddiqi, Computation of Molecular Spectra on a Quantum Processor with an Error-Resilient Algorithm, Phys. Rev. X 8, 011021 (2018).

[39] Y. Shen, X. Zhang, S. Zhang, J.-N. Zhang, M.-H. Yung, and K. Kim, Quantum implementation of the unitary coupled cluster for simulating molecular electronic structure, Phys. Rev. A 95, 020501 (2017).

[40] C. Hempel, C. Maier, J. Romero, J. McClean, T. Monz, H. Shen, P. Jurcevic, B. P. Lanyon, P. Love, and R. Babbush et al., Quantum Chemistry Calculations on a Trapped-Ion Quantum Simulator, Phys. Rev. X 8, 031022 (2018).

[41] Y. Nam, J.-S. Chen, N. C. Pisenti, K. Wright, C. Delaney, D. Maslov, K. R. Brown, S. Allen, J. M. Amini, and J. Apisdorf et al., Ground-state energy estimation of the water molecule on a trapped-ion quantum computer, Npj Quantum Inf. 6, 1 (2020).

[42] P. G. Szalay, M. Nooijen, and R. J. Bartlett, Alternative ansätze in single reference coupled-cluster theory. iii. a critical analysis of different methods, J. Chem. Phys. 103, 281 (1995).

[43] A. G. Taube and R. J. Bartlett, New perspectives on unitary coupled-cluster theory, Int. J. Quantum Chem. 106, 3393 (2006).

[44] B. Cooper and P. J. Knowles, Benchmark studies of variational, unitary and extended coupled cluster methods, J. Chem. Phys. 133, 234102 (2010).

[45] F. A. Evangelista, Alternative single-reference coupled cluster approaches for multireference problems: The simpler, the better, J. Chem. Phys. 134, 224102 (2011).

[46] G. Harsha, T. Shiozaki, and G. E. Scuseria, On the difference between variational and unitary coupled cluster theories, J. Chem. Phys. 148, 044107 (2018).

[47] M.-A. Filip and A. J. W. Thom, A stochastic approach to unitary coupled cluster, J. Chem. Phys. 153, 214106 (2020).

[48] J. Chen, H.-P. Cheng, and J. K. Freericks, Quantuminspired algorithm for the factorized form of unitary coupled cluster theory, J. Chem. Theory Comput. 17, 841 (2021).

[49] I. G. Ryabinkin, T.-C. Yen, S. N. Genin, and A. F. Izmaylov, Qubit coupled cluster method: A systematic approach to quantum chemistry on a quantum computer, J. Chem. Theory Comput. 14, 6317 (2018).

[50] P. J. Knowles, C. Hampel, and H.-J. Werner, Coupled cluster theory for high spin, open shell reference wave functions, J. Chem. Phys. 99, 5219 (1993).

[51] M. Motta, C. Sun, A. T. Tan, M. J. O'Rourke, E. Ye, A. J. Minnich, F. G. Brandão, and G. K.-L. Chan, Determining eigenstates and thermal states on a quantum computer using quantum imaginary time evolution, Nat. Phys. 16, 1 (2019).

[52] S.-N. Sun, M. Motta, R. N. Tazhigulov, A. T. Tan, G. K.-L. Chan, and A. J. Minnich, Quantum Computation of Finite-Temperature Static and Dynamical Properties of Spin Systems Using Quantum Imaginary Time Evolution, Phys. Rev. X Quantum 2, 010317 (2021).

[53] J. R. McClean, M. E. Kimchi-Schwartz, J. Carter, and W. A. de Jong, Hybrid quantum-classical hierarchy for mitigation of decoherence and determination of excited states, Phys. Rev. A 95, 042308 (2017).
[54] R. M. Parrish and P. L. McMahon, Quantum filter diagonalization: Quantum eigendecomposition without full quantum phase estimation, arXiv:1909.08925 (2019).

[55] N. H. Stair, R. Huang, and F. A. Evangelista, A multireference quantum krylov algorithm for strongly correlated electrons, J. Chem. Theory Comput. 16, 2236 (2020).

[56] W. J. Huggins, J. Lee, U. Baek, B. O'Gorman, and K. B. Whaley, A non-orthogonal variational quantum eigensolver, New J. Phys. 22, 073009 (2020).

[57] D. Wecker, M. B. Hastings, and M. Troyer, Progress towards practical quantum variational algorithms, Phys. Rev. A 92, 042303 (2015).

[58] P. Gokhale, O. Angiuli, Y. Ding, K. Gui, T. Tomesh, M. Suchara, M. Martonosi, and F. T. Chong, Minimizing state preparations in variational quantum eigensolver by partitioning into commuting families, arXiv:1907.13623 (2019).

[59] T.-C. Yen, V. Verteletskyi, and A. F. Izmaylov, Measuring all compatible operators in one series of single-qubit measurements using unitary transformations, J. Chem. Theory Comput. 16, 2400 (2020).

[60] V. Verteletskyi, T.-C. Yen, and A. F. Izmaylov, Measurement optimization in the variational quantum eigensolver using a minimum clique cover, J. Chem. Phys. 152, 124114 (2020).

[61] W. J. Huggins, J. R. McClean, N. C. Rubin, Z. Jiang, N. Wiebe, K. B. Whaley, and R. Babbush, Efficient and noise resilient measurements for quantum chemistry on near-term quantum computers, npj Quantum Inf. 7, 1 (2021).

[62] J. R. McClean, F. M. Faulstich, Q. Zhu, B. O'Gorman, Y. Qiu, S. R. White, R. Babbush, and L. Lin, Discontinuous galerkin discretization for quantum simulation of chemistry, New J. Phys. 22, 093015 (2020).

[63] M. Schuld, V. Bergholm, C. Gogolin, J. Izaac, and N. Killoran, Evaluating analytic gradients on quantum hardware, Phys. Rev. A 99, 032331 (2019).

[64] J. S. Kottmann, A. Anand, and A. Aspuru-Guzik, A feasible approach for automatically differentiable unitary coupledcluster on quantum computers, arXiv:2011.05938 (2020).

[65] I. G. Ryabinkin, R. A. Lang, S. N. Genin, and A. F. Izmaylov, Iterative qubit coupled cluster approach with efficient screening of generators, J. Chem. Theory Comput. 16, 1055 (2020).

[66] Z.-J. Zhang, T. H. Kyaw, J. S. Kottmann, M. Degroote, and A. Aspuru-Guzik, Mutual information-assisted adaptive variational quantum eigensolver ansatz construction, arXiv:2008.07553 (2020).

[67] J. Liu, Z. Li, and J. Yang, An efficient adaptive variational quantum solver of the schrodinger equation based on reduced density matrices, arXiv:2012.07047 (2020).

[68] P. K. Barkoutsos, J. F. Gonthier, I. Sokolov, N. Moll, G. Salis, A. Fuhrer, M. Ganzhorn, D. J. Egger, M. Troyer, A. Mezzacapo, S. Filipp, and I. Tavernelli, Quantum algorithms for electronic structure calculations: Particlehole hamiltonian and optimized wave-function expansions, Phys. Rev. A 98, 022322 (2018).

[69] F. A. Evangelista, G. K.-L. Chan, and G. E. Scuseria, Exact parameterization of fermionic wave functions via unitary coupled cluster theory, J. Chem. Phys. 151, 244112 (2019). 
[70] T. D. Crawford and H. F. Schaefer, An introduction to coupled cluster theory for computational chemists, Rev. Comput. Chem. 14, 33 (2000).

[71] J. Lee, W. J. Huggins, M. Head-Gordon, and K. B. Whaley, Generalized unitary coupled cluster wave functions for quantum computation, J. Chem. Theory Comput. 15, 311 (2018).

[72] T. Helgaker, P. Jørgensen, and J. Olsen, in Molecular Electronic-Structure Theory (John Wiley \& Sons, Ltd, 2000) Chap. 13, p. 648.

[73] P. Pulay, Convergence acceleration of iterative sequences. the case of SCF iteration, Chem. Phys. Lett. 73, 393 (1980).

[74] G. E. Scuseria, T. J. Lee, and H. F. Schaefer, Accelerating the convergence of the coupled-cluster approach: The use of the DIIS method, Chem. Phys. Lett. 130, 236 (1986).

[75] D. Aharonov, V. Jones, and Z. Landau, A polynomial quantum algorithm for approximating the jones polynomial, Algorithmica 55, 395 (2009).

[76] D. W. Berry, C. Gidney, M. Motta, J. R. McClean, and R. Babbush, Qubitization of arbitrary basis quantum chemistry leveraging sparsity and low rank factorization, Quantum 3, 208 (2019).

[77] J. Romero, R. Babbush, J. R. McClean, C. Hempel, P. J. Love, and A. Aspuru-Guzik, Strategies for quantum computing molecular energies using the unitary coupled cluster ansatz, Quantum Sci. Technol. 4, 014008 (2019).

[78] F. Arute, K. Arya, R. Babbush, D. Bacon, J. C. Bardin, R. Barends, S. Boixo, M. Broughton, B. B. Buckley, and D. A. Buell et al., Hartree-Fock on a superconducting qubit quantum computer, Science 369, 1084 (2020).

[79] M. Motta, D. M. Ceperley, G. K.-L. Chan, J. A. Gomez, E. Gull, S. Guo, C. A. Jiménez-Hoyos, T. N. Lan, J. Li, and F. Ma et al., Towards the Solution of the Many-Electron Problem in Real Materials: Equation of State of the Hydrogen Chain with State-Of-The-Art Many-Body Methods, Phys. Rev. X 7, 031059 (2017).

[80] M. Motta, C. Genovese, F. Ma, Z.-H. Cui, R. Sawaya, G. K.-L. Chan, N. Chepiga, P. Helms, C. Jiménez-Hoyos, and A. J. Millis et al., Ground-State Properties of the Hydrogen Chain: Dimerization, Insulator-To-Metal Transition, and Magnetic Phases, Phys. Rev. X 10, 031058 (2020).

[81] N. H. Stair and F. A. Evangelista, Exploring Hilbert space on a budget: Novel benchmark set and performance metric for testing electronic structure methods in the regime of strong correlation, J. Chem. Phys. 153, 104108 (2020).

[82] D. G. Smith, L. A. Burns, A. C. Simmonett, R. M. Parrish, M. C. Schieber, R. Galvelis, P. Kraus, H. Kruse, R. Di Remigio, and A. Alenaizan et al., Psi4 1.4: Open-source software for high-throughput quantum chemistry, J. Chem. Phys. 152, 184108 (2020).

[83] N. H. Stair and F. A. Evangelista, QForte: A quantum computer simulator and algorithms library for molecular simulation (see https://github.com/evangelistalab/qforte and https://education.molssi.org/2020-software-fellow-posters/ nick-stair), (2020).

[84] C. G. Broyden, The convergence of a class of doublerank minimization algorithms: 2 . the new algorithm, J. Inst. Math. Appl. 6, 222 (1970).

[85] R. Fletcher, A new approach to variable metric algorithms, Comput. J 13, 317 (1970).

[86] D. Goldfarb, A family of variable-metric methods derived by variational means, Math. Comput. 24, 23 (1970).

[87] D. F. Shanno, Conditioning of quasi-newton methods for function minimization, Math. Comput. 24, 647 (1970).

[88] P. Virtanen, R. Gommers, T. E. Oliphant, M. Haberland, T. Reddy, D. Cournapeau, E. Burovski, P. Peterson, W. Weckesser, and J. Bright et al., Scipy 1.0: Fundamental algorithms for scientific computing in python, Nat. Methods 17, 261 (2020).

[89] M. B. Hastings, D. Wecker, B. Bauer, and M. Troyer, Improving quantum algorithms for quantum chemistry, Quantum Info. Comput. 15, 1 (2015).

[90] J. B. Schriber and F. A. Evangelista, Communication: An adaptive configuration interaction approach for strongly correlated electrons with tunable accuracy, J. Chem. Phys. 144, 161106 (2016).

[91] G. D. Purvis III and R. J. Bartlett, A full coupled-cluster singles and doubles model: The inclusion of disconnected triples, J. Chem. Phys. 76, 1910 (1982).

[92] K. Raghavachari, G. W. Trucks, J. A. Pople, and M. Head-Gordon, A fifth-order perturbation comparison of electron correlation theories, Chem. Phys. Lett. 157, 479 (1989).

[93] U. S. Mahapatra, B. Datta, and D. Mukherjee, A sizeconsistent state-specific multireference coupled cluster theory: Formal developments and molecular applications, J. Chem. Phys. 110, 6171 (1999).

[94] F. A. Evangelista, W. D. Allen, and H. F. Schaefer, Coupling term derivation and general implementation of statespecific multireference coupled cluster theories, J. Chem. Phys. 127, 024102 (2007).

[95] F. A. Evangelista, E. Prochnow, J. Gauss, and H. F. Schaefer, Perturbative triples corrections in state-specific multireference coupled cluster theory, J. Chem. Phys. 132, 074107 (2010).

[96] K. Setia, R. Chen, J. E. Rice, A. Mezzacapo, M. Pistoia, and J. D. Whitfield, Reducing qubit requirements for quantum simulations using molecular point group symmetries, J. Chem. Theory Comput. 16, 6091 (2020).

[97] V. Giovannetti, S. Lloyd, and L. Maccone, Quantum Random Access Memory, Phys. Rev. Lett. 100, 160501 (2008).

[98] S. Lloyd, M. Mohseni, and P. Rebentrost, Quantum principal component analysis, Nat. Phys. 10, 631 (2014).

[99] R. LaRose, A. Tikku, É. O’Neel-Judy, L. Cincio, and P. J. Coles, Variational quantum state diagonalization, npj Quantum Inf. 5, 1 (2019). 